Gerión. Revista de Historia Antigua

ISSN: 0213-0181

http://dx.doi.org/10.5209/GERI.56956

\title{
Minerva ed i pueri: proposta per una rilettura di alcune fonti letterarie
}

\author{
Tiziano Cinaglia ${ }^{1}$
}

Recibido: 23 de mayo de 2016 / Aceptado: 15 de noviembre de 2016

Riassunto. La relazione che intercorre tra Minerva ed i pueri romani, nota archeologicamente dai più antichi santuari italici della dea ed inoltre documentata dalla presenza dell'altare della dea Iuventas nella cella di Minerva del tempio capitolino, appare altresì evidente ad una più approfondita disamina di alcune fonti letterarie di epoca classica; in particolare, risultano assolutamente determinanti le testimonianze relative alla prassi del tributo del minerval, alla tutela della dea sulla memoria e, infine, le numerose attestazioni del patronato di Minerva sulle attività dell'artigianato tessile. Quantunque pertinenti ad ambiti totalmente diversi, tali competenze di Minerva sono collegate da un unico filo conduttore, riconducibili ad una origine comune costituita dal primigenio culto tributatole come dea tutelare dei riti di passaggio post-puberali.

Parole chiave: Minerva; pueritia; Minerval; Dea della memoria; lanificium; riti di passaggio.

\section{[en] Minerva and the Pueri: reconsidering some Literary Sources}

Abstract. The connection between Minerva and Roman pueri, well known archaeologically in the oldest Italic shrines of the goddess and documented by the presence of goddess Iuventas in the cella of Minerva of the Capitoline temple, can be also clearly revealed by a more detailed examination of some ancient sources; in this regard, the literary testimony concerning the practice of minerval, the protection of the goddess on the memory and, lastly, the numerous proofs of Minerva's patronage on textile handicraft activities are absolutely crucial. Although pertaining to undoubtedly very different fields, such competences of the goddess are reciprocally linked by a common thread, all of them being ascribable to a common genesis originating from the primitive cult of Minerva as tutelary goddess of post-puberty transitional rites.

Keywords: Minerva; Pueritia; Minerval; Goddess of memory; Lanificium; Transitional rites.

Sommario. 1. Il minervale munus. 2. Minerva dea della memoria. 3. Minerva dea lanificii. 4. Conclusioni. 5. Bibliografia.

Cómo citar: Cinaglia, T. (2017) Minerva ed i pueri: proposta per una rilettura di alcune fonti letterarie, en Gerión 35/1, 75-98.

\footnotetext{
1 Dottore di Ricerca dell'Università degli Studi di Perugia

E-mail: tiziano.cinaglia@virgilio.it
} 
Minerva era, per gli autori antichi, indubbiamente una divinità poliedrica, dalle mille sfaccettature; una dea il cui patronato si estendeva alle attività più varie ed eterogenee e la cui sfera di competenza andava dall'ambito militare a quello medico, da quello poliadico a quello artistico-letterario. Mille dea est operum: è così, in effetti, che Ovidio, nel celeberrimo brano del III libro dei Fasti, definisce Minerva: ${ }^{2}$ una divinità dalle mille arti. Sicuramente, tanta parte nella creazione di un quadro generale privo di univocità per la figura di Minerva ebbe la stessa tradizione letteraria latina che, attingendo a piene mani dai modelli greci, intervenne inevitabilmente, alterandole, anche sulle caratteristiche originarie della dea. Però, ancora in epoca tardo-repubblicana ed imperiale, anche nelle fonti si possono rintracciare, seppur velati da una patina grecizzante, i connotati della primigenia natura di Minerva: un fil rouge che collega più testimonianze, all'apparenza molto dissimili tra loro, e che ci riconduce a quella che doveva essere la dea delle origini.

\section{Il minervale munus}

I primi documenti che prenderemo in considerazione sono quelli pertinenti al 'dono minervale', che la vulgata moderna genericamente definisce come il pagamento che gli scolari effettuavano nei confronti dei loro maestri nel giorno delle Quinquatrus del 19 marzo, ovvero nel giorno del dies natalis di Minerva: ${ }^{3}$ minerval o minervale munus, per l'appunto, sono i termini con cui le fonti indicano questo tributo. Le questioni che pone la Dubosc nell'introduzione al suo articolo sul presunto nesso tra Minerva ed il denaro, teorizzato proprio sulla base dell'esistenza di questo 'tributo minervale', hanno l'indubbio merito di indirizzare l'attenzione su di un aspetto centrale della ritualità della religione romana, ovvero la nascita e la funzione del rituale stesso: ${ }^{4}$ troppo spesso, infatti, l'analisi di storici e storici delle religioni si focalizza semplicisticamente sull'esteriorità del rito, dimenticandosi però di risalire piuttosto alle origini di quella prassi, di quei gesti ripetuti e fossilizzatisi nel tempo, indagandone il contesto storico e sociale ed il valore che veniva loro attribuito. Pertanto, evitando ulteriori divagazioni, dobbiamo riconoscere che tra i vari interrogativi che l'autrice ci propone, due in particolare hanno un ruolo centrale ai fini di questa analisi: il primo quesito, invero, ci pone di fronte alla necessità di indagare le motivazioni sulle quali si fonda il dono in denaro alla dea; il secondo, invece, ancor più fondamentale, rivolge l'attenzione all'individuazione delle origini storiche di questa pratica; ossia, in buona sostanza, dobbiamo chiederci: perché esisteva questa offerta di denaro? ed inoltre, come e quando nacque questa prassi

\footnotetext{
Ov. Fast. 3.833 .

Ov. Fast. 3.809-812: Una dies media est et fiunt sacra Minervae / nominaque a iunctis quinque diebus habent. / Sanguine prima vacat nec fas concurrere ferro; / causa, quod est illa nata Minerva die. Della pertinenza di questa festività a Minerva siamo documentati, inoltre, anche dalle indicazioni dell'annalistica romana che, sin dal calendario pre-cesariano, attribuiscono le Quinquatrus alla nostra dea: i Fasti Antiates Maiores riportano la dicitura QVIN Minervae (DEGRASSI, Inscr. It., XIII.2, 7), mentre i Fasti Farnesiani riportano la dicitura QVIN Minerv[ae] (DEGRASSI, Inscr. It., XIII.2, 225); infine, Verrio Flacco, nel commento ai Fasti Praenestini, dopo la dicitura $[Q V I N] Q \cdot N$, aggiunge: recte tamen alii putarunt dictum ab eo quod hic dies est post diem u idus quod - in Latio · post idus dies simili ratione declinarentur / artificum dies quod Minervae aedis · in Aventino $\cdot$ eo $\cdot$ die $\cdot$ est dedicata / Salii faciunt $\cdot$ in comitio $\cdot$ saltu adstantibus pontificibus $\cdot$ et $\cdot$ trib $\cdot$ celer (DEGRASSI, Inscr. It . XIII.2, 122s.).

4 Dubosc 2002, 383.
} 
devozionale? Le fonti sul tema del minerval sono notevoli, sia numericamente che per puntualità e quantità delle informazioni, e ci permettono pertanto una disamina molto circostanziata ed attendibile.

Il primo autore a nominare il 'dono minervale' è Varrone, il quale, imbastendo nel terzo libro del De Re Rustica un lungo dialogo tra il senatore Q. Axius, l'augure A. Claudius Pulcher e L. Cornelius Merula sull'allevamento in villa (villatica pastio), ${ }^{5}$ fa dire al primo dei tre che si sarebbe impegnato ad istruire Merula in tale attività in cambio del pagamento di un minerval. ${ }^{6}$ La seconda testimonianza, a distanza di un secolo e mezzo da quella dell'erudito reatino, è quella di Giovenale, all'interno del decimo libro delle Saturae; ma se nel brano di Varrone il minerval si delineava come un pagamento in cambio di una istruzione impartita, o comunque dell'acquisizione di una professionalità, per il retore romano invece questo tributo diventa chiaramente un dono, un obolo che rappresentava il segno della devozione da parte dello scolaro nei confronti di Minerva, un'offerta da realizzarsi proprio durante la festività delle Quinquatrus. ${ }^{7}$ Una medesima interpretazione del significato simbolico del minerval, inoltre, la si ricava altresì da due ulteriori fonti, ovvero quelle dei Padri della Chiesa san Girolamo e Tertulliano: quest'ultimo, infatti, afferma esplicitamente che il primo pagamento ricevuto dai maestri di scuola altro non è che una forma di gratitudine da parte dello studente nei confronti della dea, un tributo consacrato Minervae et honori et nomini $; 8$ per quanto concerne la testimonianza di San Girolamo, da ultimo, si deve riconoscere che se da un lato il minerval torna ad essere associato alla riscossione di un pagamento da parte del maestro -con esempi assolutamente esecrabili dell'impiego di questo denaro, un leitmotiv ricorrente nella letteratura patristica, allo scopo di condannare l'eresia della religione pagana- d'altro canto, però, questo tributo è altresì caratterizzato come un obolo da dedicare in temple stipes, quindi nuovamente presentato come un dono da consacrare alla dea. ${ }^{9}$ L'ultima fonte relativa a questa prassi -anche se nel brano non si fa esplicita menzione del minerval- è quella di Macrobio, il quale ci tramanda che nel mese di marzo i maestri ricevevano il compenso per l'anno precedente: è indubbiamente più che probabile, anche se non verificabile, che questo pagamento coincidesse con la festa delle Quinquatrus e che si trattasse, per l'appunto, del minerval, anche se non esplicitamente menzionato. ${ }^{10}$ Ma a completare il quadro sulla effettiva valenza del munus minervalis e sul contesto in cui questo tributo in denaro veniva corrisposto, o piuttosto versato, dobbiamo inoltre considerare due ultimi passaggi, il primo tratto dagli scritti di Orazio, l'altro da Simmaco, nei quali si descrive l'atmosfera che regnava durante la festività delle Quinquatrus: ${ }^{11}$ sia il poeta venusino sia il celebre oratore romano, infatti, ci

Più in dettaglio su questi personaggi vd. GuIRARD 1997, introduzione.

6 Varro RR 3.2.18: Axius: 'Merula mi-inquit-recipe me quaeso discipulum villaticae pastionis'. -Ille: 'Quin simulac promiseris minerval, incipiam'-inquit.

7 Iuv. Sat. 10.114-117: Eloquium ac famam Demosthenis aut Ciceronis / incipit optare et totis quinquatribus optat / quisquis adhuc uno parcam colit asse Minervam, / quem sequitur custos angustae vernula capsae.

8 Tert. De idol. 10.1: Quis ludimagister sine tabula septem idolorum Quinquatria tamen frequentabit? Ipsam primam novi discipuli stipem Minervae et honori et nomini consecrat.

9 Hieron. Ep. in Ephes. 4.4: Minervale munus <quod $>$ grammaticus et orator aut in sumptos domesticos, aut in temple stipes, aut in sordida scorta convertit.

10 Macr. Sat. 1.12.7: Hoc mense mercedes exolvebant magistris quas conpletus annus deberi fecit.

11 Hor. Ep. 2.2.195-198: Distat enim, spargas tua prodigus an neque sumptum / Invitus facias neque plura parare labores, / Ac potius, puer ut festis Quinquatribus olim, / Exiguo gratoque fruaris tempore raptim; Symm. Ep. 5.85.3: Quod superest, oro iam venias et praesentia tua aurea honorem festorum dierum. Nempe Minervae tibi 
testimoniano, nonostante la distanza di secoli che intercorre tra i due, come quei giorni siano sempre stati in realtà vissuti, e quindi in seguito nostalgicamente ricordati, come una festa degli studenti, e non invece come un giorno festivo dei vituperati e sottopagati maestri. ${ }^{12}$

Che fossero gli scolari a festeggiare il 19 marzo non è una considerazione di poco conto, poiché è sicuramente un indizio inconfutabile del ruolo di primo piano giocato dai fanciulli in questa giornata; se si aggiunge, per di più, che erano sempre gli studenti a consacrare il minerval alla dea per mezzo dei loro precettori, si deve ammettere, conseguentemente, che il rapporto sotteso a questa celebrazione fosse quello tra Minerva e gli scolari, e non tra la dea e gli insegnanti. ${ }^{13}$ Ma questa relazione privilegiata tra Minerva e gli studenti, tra Minerva ed i giovani, è rimarcata da un ben più noto brano nel panorama della letteratura latina, ovvero a quello del terzo libro dei Fasti di Ovidio, dedicato per l'appunto alla dea ed alla festività delle Quinquatrus: il poeta di Sulmona, infatti, dopo un breve esordio in cui centra l'attenzione sull'origine del nome della festa e sulla descrizione delle attività e delle celebrazioni ad essa connesse, esattamente nell'incipit della sua invocazione alla dea si rivolge in primo luogo ad un uditorio rappresentato proprio da pueri teneraeque puellae, affinché costoro preghino convenientemente Pallade-Minerva, poiché solo chi avrebbe avuto la benevolenza della dea sarebbe divenuto doctus, ovvero bene istruito. ${ }^{14}$ La collocazione in positio princeps del riferimento ai fanciulli romani tra i cinquanta versi dedicati a Minerva, a mio parere, non può essere priva di significato, né tanto meno casuale: reputo sia innegabile, dunque, ammettere che, se le Quinquatrus costituivano una festa degli scolari, come testimoniato dalle fonti, la scelta di Ovidio di rivolgersi dapprima ai fanciulli dovesse risiedere nel legame tra questi ultimi e Minerva. Quindi, per tornare al minerval, ritengo che vi si debba coerentemente vedere un dono da parte degli studenti per ingraziarsi la divinità che vegliava sulla loro istruzione, ovvero un'offerta sacra alla dea, e non un generico pagamento ai precettori scolastici; ma, d'altronde, il nome stesso 'minerval' indica esplicitamente a chi fosse diretto tale tributo in denaro: infatti, chi, se non Minerva, poteva essere il destinatario di 'una cosa pertinente a Minerva' ${ }^{15}$ L'attribuzione del minerval a Minerva non è certamente solo di natura etimologica, non è un mero fatto linguistico, ma presuppone evidentemente anche una pertinenza di matrice cultuale del dono alla dea; ora, chiarito e ribadito ciò, perché dunque, nell'ipotesi che si trattasse di un compenso per i maestri, avrebbe dovuto essere denominato con siffatto sostantivo? perché definirlo come una cosa di proprietà della dea, se ella concretamente non prendeva parte alcuna in questa gratifica/compenso? E perché inoltre, invertendo il punto di vista, i maestri avrebbero goduto anticamente di così

sollemne de scholis notum est, ut fere memores sumus etiam procedente aevo puerilium feriarum.

12 Hild 1969c, 802s.; Frasca 1996, 521ss.

13 Che il minerval potesse essere diventato, già in età tardo-repubblicana, parte del corrispettivo da pagare agli insegnanti, come d'altronde sostengono vari studiosi, non è in discussione (HILD 1969c, 802; BonNER 1977, 149; Guirard 1997, 64; SChilling 2003, nota 239). Ma, concordando con quanto affermato dalla Granino Cecere, non si può semplicisticamente identificare il minerval con il compenso dovuto ai precettori scolastici (GrANINo CECERE 2001, 27, nota 2): in effetti, il collegamento è con gli studenti e non con i maestri, dovendo riconoscere nelle Quinquatrus una festa degli scolari, come dicono chiaramente le fonti e come sostiene parte della vulgata moderna (Fougères 1969, 1929; FraZer 1976, 182; SCullard 1981, 93).

14 Ov. Fast. 3.815-816: Pallada nunc pueri teneraeque orate puellae! / Qui bene placarit Pallada, doctus erit.

15 Minerval, -alis, sostantivo della terza declinazione, indica ciò che è proprio di Minerva, ciò che è pertinente a Minerva, come il corrispettivo aggettivo della seconda classe minervalis, $-e$. 
scarsa reputazione ed oltretutto di così miseri ed incerti salari, se effettivamente nel corso della festività delle Quinquatrus fosse stata garantita alla loro categoria addirittura la protezione divina di Minerva e per di più anche una retribuzione sicura -ed in un certo qual modo sacra-, se nel minerval si deve effettivamente riconoscere il loro compenso? ${ }^{16}$

Credo sia dunque inevitabile ammettere che, per lo meno originariamente, il minervale munus altro non fosse che un dono, per l'appunto, destinato a Minerva, anzi 'di proprietà di Minerva'; dunque, un atto di venerazione tributato alla dea da parte degli studenti, tramutatosi solo successivamente in pagamento per la loro educazione ed istruzione e, quindi, anche in un compenso, o in parte di esso, per gli stessi precettori. Non possiamo stabilire se la pratica di questa offerta in denaro risalga sin alle origini del culto di Minerva; se così fosse, dovremmo però certamente ipotizzare altre forme ed altre circostanze rispetto a quelle dell'educazione scolastica, dell'istruzione tout court: ${ }^{17}$ infatti, come è noto, la prima scuola a pagamento a Roma verrà istituita soltanto nella seconda metà del III secolo a.C., per l'annalistica romana ad opera di un liberto di Spurius Carvilius. ${ }^{18} \mathrm{Si}$ deve necessariamente concludere, pertanto, che la prassi del minervale munus, nelle forme descritteci dalle fonti, si sia sviluppata in un momento in cui l'istituzione scolare avesse raggiunto una certa diffusione e sistematicità, ovvero verosimilmente non prima del II sec. a.C. Ma se 1'esistenza del minerval per il periodo arcaico non è attestata né dimostrabile, è invece indubbia l'arcaicità del nesso tra Minerva ed i fanciulli romani, riconducibile sino agli albori della storia di Roma: la presenza di un altare dedicato alla dea Iuventas all'interno della cella di Minerva nel tempio capitolino, infatti, sottolinea per l'appunto quanto stretto e quanto risalente fosse questo legame; ${ }^{19}$ la dea Iuventas,

16 La letteratura moderna è concorde nell'attribuire a Minerva il patronato sui maestri (vd. nota 13 ed inoltre anche Girard 1981, 209; Pedroni 1998, 48), un ruolo che, d'altro canto, si può facilmente desumere dai due versi di Ovidio all'interno del brano sulle Quinquatrus (Fast. 3.829-830: Nec vos, turba fere censu fraudata, magistri, / spernite; discipulos attrahit illa novos); ma proprio questi versi dimostrano, per di più, come il lavoro del precettore avesse scarsa considerazione, aggiungendo che costoro venivano spesso anche frodati: evidentemente, la tutela da parte di Minerva sulla categoria dei magistri non doveva poi essere così 'efficace'.

17 Ma non vi è alcuna necessità di ipotizzare, come fa Bonner, un'offerta 'in natura' per il periodo arcaico; il passo di Varrone ( $R R$ 3.2.18 - vd. nota 4) che lo storico inglese apporta come testimonianza non ci documenta il alcun modo di questo pagamento "in kind" (Bonner 1977, 149): il dialogo tra Axius, Appius e Merula, una scenetta che possiamo definire dai toni comici, rivela soltanto che il primo dei personaggi appena citati garantisce come forma di corresponsione per l'apprendimento della villatica pastio o un pagamento in denaro o l'equivalente in animali. Non vi è nulla, in questa situazione, che possa richiamare il contesto o l'ambientazione delle Quinquatrus, né tanto meno si possono riconoscere le caratteristiche precipue del minerval nel pagamento effettuato col bestiame; ancor più astrusa, pertanto, in quanto priva di qualsiasi fondamento documentario, è l'idea che questa pratica del 'pagamento in natura come minerval' fosse radicata nell' antichità e caduta in disuso solo nella tarda repubblica.

18 Spurius Carvilius, console due volte nel 234 e nel 228 a.C. (MRR I, 223s.; 228s.), sarebbe collegato alla conquista di Falerii ed in qualche modo in relazione con l'episodio miti-storico del tentativo di un maestro di scuola della capitale falisca di tradire la propria gente consegnando gli scolari ai Romani durante l'assedio del 394 a.C. (Liv. 5.27); la nascita di questa prima scuola romana si collocherebbe, dunque, grosso modo nel decennio dal 240 al 230, ovvero nello stesso periodo in cui ha origine la letteratura latina con l'opera di Livio Andronico (Girard 1989, 168s.; Pedroni 1998, 44ss.).

19 Alla dea Iuventas, infatti, in epoca storica, erano consacrati una aedicula ed una ara dentro la struttura templare capitolina, più precisamente all'interno della cella di Minerva (Plin. HN 35.108). L'origine stessa del culto della dea Iuventas è innegabilmente molto risalente, dal momento che l'antiquaria romana ne parla in relazione alla nota leggenda sulla costruzione del Capitolium stesso: infatti, secondo la tradizione, al momento dell'exauguratio, ossia della liberazione dell'area dalla presenza di altre divinità, gli augures non ottennero di poter spostare tutti i culti presenti sul colle del Campidoglio, poiché in tre si opposero, ossia Marte, Terminus e Iuventas (Liv. 5.55.7; D.H. 3.69.3-5); è indubbio, pertanto, che la tradizione individuasse in queste divinità 
invero, era la dea novorum togatorum, colei che certificava il cambiamento di status dei giovani romani, tenuti al pagamento rituale di un tributo alla dea al momento del passaggio dall'adolescenza all'età adulta. ${ }^{20}$ Ancora una volta, dunque, nell'esaminare il culto di Minerva, e di riflesso il significato del minerval nella sua celebrazione, appare fondante il nesso tra la dea e l'adolescenza, tra la dea ed i pueri romani; proprio in virtù di questa relazione, pertanto, ritengo si debba leggere la prassi del minerval, come una forma di devozione ritualizzata da parte degli adolescenti nei confronti della dea che vegliava sulla loro crescita e sulla loro formazione. ${ }^{21}$

Tornando al presunto rapporto tra Minerva ed il denaro teorizzato dalla Dubosc, si deve pertanto definitivamente escludere che la pratica del 'dono minervale' possa essere di supporto a tale interpretazione; ma, in realtà, ad un rapido esame delle altre prove elencate a sostegno di suddetta relazione, vediamo come anche queste siano tutte inevitabilmente da cassare, confutando definitivamente la tesi di una Minerva 'ricca'. In effetti, che anche i medici potessero seguire una simile prassi presentando un'offerta alla dea, come sostiene l'autrice, è assolutamente indimostrabile; ${ }^{22} \mathrm{i}$ versi del terzo libro dei Fasti, addotti a supporto di questa ipotesi, non ci suggeriscono invero nulla del genere, poiché il poeta invita i medici soltanto a donare genericamente munera de vestris pauca: ${ }^{23}$ in questi 'pochi doni' da offrire alla dea possiamo vedervi qualsiasi tipo di offerta, anche -ma non necessariamente- in denaro; in ogni caso, il distico in questione non denota alcuna particolare venerazione da parte della categoria: infatti, l'esistenza del tempio di Minerva Medica sull'Esquilino non è certamente una testimonianza a favore di una particolare devozione dei medici nei confronti della divinità, come lascia invece intendere l'autrice, ${ }^{24}$ bensì la semplice documentazione di un culto di carattere salutare riservato alla dea, culto attestato archeologicamente a partire dal IV secolo a.C. ed al quale si rivolgevano, evidentemente, i malati della comunità cittadina per ottenere la guarigione -come oltretutto dimostrato dai

dei culti fondanti della religione romana, risultando questi inamovibili anche di fronte al nascente principale culto statale. La centralità del culto di Iuventas nella Roma arcaica è dunque un patente indizio dell'importanza che anticamente rivestiva il periodico rinnovamento della comunità mediante l'annuale inserimento dei nuovi iuvenes, garantiti dalla dea insieme a Minerva, alla presenza degli dei poliadici (sulla dea Iuventas vd. HiLD 1969a, 785s; sul concetto di iuvenes vd. ZIEBARTH 1919, 1358; JULLIAN 1969, 782ss.).

20 La dea Iuventas è così definita da Sant'Agostino (Civ. 4.11: quae post praetextam excipiat iuvenalis aetatis exordia), Servio (Aen. 4.32: iuventa autem pro iuventate accipiendum: nam 'Iuventa' dea illius aetatis est, 'iuventas' aetas ipsa iuvenilis, 'iuventus' iuvenum multitudo) e Tertulliano (ad Nat. 2.11: dea novorum togatorum); secondo la tradizione, il rituale del pagamento di un tributo da parte del giovane sarebbe stato istituito da Servio Tullio, ad ulteriore dimostrazione dell'arcaicità di questa pratica (D.H. 4.15.5). Come afferma Montanari, pertanto, "l'importanza che la dea Iuventas assumeva nel passaggio da un «insieme» all'altro", da una classe di età all’altra, rimonta indubitabilmente all’età arcaica (MONTANARI 1988, 130).

21 È forse ascrivibile alla relazione tra Minerva e l'educazione dei pueri la costruzione, in epoca imperiale, della sede di un paedagogium nelle immediate vicinanze dell'arcaico tempio di Minerva Capta sul Celio, a poche centinaia di metri da esso, lungo il vicus Capitis Africae: in questa struttura operavano i paedagogi puerorum (CIL VI 1052 e 8982-8985), ai quali presumibilmente competeva l'addestramento dei valletti imperiali, impiegati in compiti generali di spettacolo e celebrazione (VALENTINI - ZuCCHETTI 1940-41, I, 93, nota 4; Platner - Ashby 1965, 98s.; Giannelli 1993, 211; Pavolini 1993, 235). La vicinanza tra il paedagogium, individuabile nell'area dell'attuale Piazza Celimontana, ed il Minervium, collocabile nella zona della chiesa dei SS. Quattro Coronati (Varr. Ling. Lat. 5.47; Ov. Fast. 3.835-837. Colini 1944, 39; PlatNer - Ashby 1965, 344; JordAN - HuElSEN, 1970-71, I.3, 226; SchürmANn 1985, 10; CoARElli 1996, 255; Id. 1997-98, 210), può dunque costituire un ulteriore, seppur labile, indizio del nesso Minerva-pueritia.

22 Dubosc 2002, 381ss.

23 Ov. Fast. 3.827-828: Vos quoque, Phoebea morbos qui pellitis arte, / munera de vestris pauca referte deae.

24 Dubosc 2002, 385. Anche Fougères e Cèbe sono, erroneamente, di questo parere (FougÈres 1969, 1929; CÈBE 1996, 1810). 
numerosi ex-voto pertinenti a questo santuario. ${ }^{25}$ L'unico altro, seppur labile, indizio del fatto che i medici prendessero parte ai festeggiamenti del 19 marzo è una satura di Varrone, intitolata appunto Quinquatrus e nella quale uno dei personaggi messi in scena è proprio un medico ${ }^{26}$ ma l'esiguità del numero dei frammenti giunti sino a noi non ci permette di stabilire quale ruolo effettivamente giocasse il medico in questa vicenda dal tono serio-comico e quale fosse, eventualmente, il suo rapporto con la festa. Se, dunque, rimane indiscussa la devozione di coloro che praticano l'ars Phoebea, come dice Ovidio, nulla ci autorizza invece ad ipotizzare che costoro pagassero un qualsiasi tributo specifico alla dea.

$\mathrm{Ma}$, a ragion del vero, si deve riconoscere che anche la successiva testimonianza letteraria addotta come prova dalla Dubosc è assolutamente da respingere, essendo fondata su di una totale decontestualizzazione del passo indagato: 1'autrice, in effetti, ritiene di poter individuare nelle parole di Marziale la prova della 'ricchezza' di Minerva, in quanto il suo forziere habet nummos e la dea fenerat omnes deos, ${ }^{27}$ il testo, avulso dal resto del componimento poetico, può in effetti farci supporre una certa predilezione di Minerva verso il denaro. Ma in realtà, ad un sguardo più attento, $i$ versi in esame indicano semplicemente, attraverso una serie di metafore una delle quali è per l'appunto quella di Minerva che presta soldi alle altre divinità-, che la professione dell'avvocato risultava più redditizia di quella del poeta; il nesso Minerva-arte retorica è inoltre ribadito da Marziale anche in un successivo passo: $:^{28}$ infatti, in tale epigramma il poeta instaura un confronto tra Apollo e Minerva, il primo come divinità tutelare dell'arte poetica e la seconda, al contrario, a prendere le parti dell'avvocatura; in questo 'scontro' tra le due professioni, successo e denaro arridono a chi svolge il mestiere di avvocato e non al poeta, perché Romanum forum divitius est, come conclude Marziale poco più avanti nel componimento: ${ }^{29}$ non è pertanto necessario sostenere che $\mathrm{i}$ santuari di Minerva fossero talmente ricchi da poter prestare denaro all'intera classe sacerdotale adibita al culto delle restanti divinità del pantheon romano e, pertanto, individuare in suddetta metafora gli estremi di un rapporto privilegiato tra la dea ed il denaro. Minerva è 'abbiente' in

25 Platner - Ashby 1965, 344s.; Jordan - Huelsen 1970-71, I.3, 352s.; Gatti lo GuZzo 1978; Richardson 1992, 256. Nel 1887 furono scoperte in via Carlo Botta delle favissae contenenti diverse centinaia di oggetti votivi, tra i quali una lucerna iscritta col nome di Minerva ([Me]nerva dono de[det]); oltre a ciò furono rinvenute numerose statuette e testine della dea e numerosi fittili relativi ad una divinità salutare. Gli oggetti votivi rinvenuti, soprattutto ex-voto anatomici, mostrano che il santuario era dedicato ad Apollo e a Minerva nella loro veste di divinità salutari; ma questi votivi mostrano anche come questo fosse un posto abbastanza umile e modesto, non essendo stati rinvenuti oggetti di pregio.

26 Varro Sat.Men. 75, Quinquatrus (frg. 440-448). La trama, ricostruibile a grandi linee, vede due amici coinvolti in un banchetto a casa di un medico; qui si intavolerà un dibattito sugli accorgimenti da seguire per avere una vita sana, dialogo poi condito da una 'tirata' contro la medicina greca (CÈBE 1996, 1811s.).

Alcuni studiosi hanno interpretato il passo ciceroniano del De Divinatione (2.123: et si sine medico medicinam dabit Minerva ...) come prova del legame tra Minerva ed i medici (Fougères 1969, 1929; DuBOSC 2002, 385); ma in realtà questa espressione costituisce null'altro che una testimonianza della valenza salutare che poteva avere il culto della dea, e di certo non della venerazione che i medici le avrebbero tributato.

27 Mart. 1.76.5-6: Quid petis a Phoebo? Nummos habet arca Minervae; / haec sapit, haec omnes fenerat una deos.

28 Mart. 10.20.14-17: Totos dat tetricae dies Minervae / dum centum studet auribus virorum / hoc quod saecula posterique possint / Arpinis quoque conparare chartis.

29 Mart. 1.76.12: Romanum propius divitiusque forum est. Tutto il componimento è interamente costruito su questa opposizione/confronto tra la professione del poeta e quella dell'avvocato; così, dopo la metafora dell'arca, ne troviamo una seconda di carattere vegetale, in cui le nere fronde dell'albero di Minerva coprono l'edera di Apollo, l'avvocatura oscurando la più debole e povera arte poetica: vv. 7-8: Quid possunt hederae Bacchi dare? Pallados arbor / inclinat varias pondere nigra comas. 
quanto dea-avvocato, e non in quanto privilegiata destinataria di ricche offerte in denaro. Altrettanto irrilevante ed assolutamente non probante, infine, è il fatto che al momento della ricostruzione del Capitolium sotto Vespasiano siano stati gettati dei frammenti di oro e argento nelle fondazioni del tempio: $:^{30}$ nulla mette in relazione diretta ed esclusiva con Minerva questi metalli preziosi, essendo al contrario evidentemente consacrati alla triade capitolina nel suo complesso. ${ }^{31}$

\section{Minerva dea della memoria}

Minerva, pertanto, non aveva alcuna particolare 'affezione' verso il denaro. Al contrario, il 'dono minervale', come abbiamo visto, aveva tutt'altro significato: esso, in effetti, denota con chiarezza come i fanciulli romani si rivolgessero a Minerva per ottenere la sua protezione nel corso della fase di formazione dell'infanzia e dell'adolescenza, un periodo coincidente con quello dell'istruzione scolastica. Ora, sulla scorta di questa evidente e stretta relazione individuata nelle fonti letterarie, ritengo si debba riesaminare anche il ruolo di Minerva come dea della memoria propostoci dagli autori latini.

Infatti, per quanto possa sembrare inverosimile, nessuna tra le fonti conservateci definisce Minerva come dea della mens, immagine invece ampiamente difussa nella communis opinio; in realtà ci si riferiva a Minerva semplicemente, e più 'banalmente', nei termini di dea della memoria. ${ }^{32}$ Ed è proprio questa sua caratteristica, questa sua competenza, ad indirizzare erroneamente uno dei numerosi tentativi di ricostruzione etimologica del nome della dea elaborato dagli autori latini: in effetti, Arnobio, nella sua opera apologetica Adversus nationes -per la quale usa probabilmente il testo

Tac. Hist. 4.53 .

31 Dubosc 2002, 391. Da ultimo, si deve confutare recisamente anche una ulteriore affermazione della studiosa francese: infatti, l'ipotesi che si potessero fare delle offerte in denaro anche durante le Quinquatrus minusculae del 13 giugno è priva di qualsiasi base documentaria e del tutto fantasiosa, dal momento che nessuno degli elementi noti per questa festività, nessuna delle varie fonti in merito lasciano anche soltanto supporre simili atti di devozione nei confronti di Minerva per le idi di giugno (Dubosc 2002, 384); le testimonianze letterarie a riguardo, come dicevamo, sono infatti numerose e la descrizione dell'evento è essenzialmente univoca: si tratta della nota vicenda dell'esilio volontario dei tibicines a Tivoli, come forma di protesta nei confronti del senato che aveva drasticamente ridotto i loro privilegi, episodio datato al 311 a.C. e terminato con il reintegro dei flautisti nel corpo sacerdotale cittadino con le proprie originarie prerogative (Var. Ling. Lat. 4.17; Liv. 9.30.5-10; Ov. Fast. 6.651-710; Quint. Inst. 5.11.9; Plu. Quaest.Rom. 55; Fest. 134L; Cens. 12.2; Vir.Ill. 34.1).

32 In effetti, l'antiquaria romana si indirizza a Minerva come dea dell'ingenium (Ov. Fast. 3.839-40: Capitale vocamus / ingenium sollers: ingeniosa dea est; Serv. Buc. 2.61: sed quia dea est artium et ingenii, ideo ista finguntur; Isid. Or. 8.11.71: Hanc enim inventricem multorum ingeniorum perhibent, et inde eam artem et rationem interpretantur), o piuttosto come dea della sapientia (Paul.Fest. 109L: Minerva dicta, quod bene moneat. Hanc enim pagani pro sapientia ponebant; Serv. Aen. 2.615: ut Pallas quas condidit arces, et quia summa quaeque possidet sapientia) o infine come un connubio tra le due sfere di pertinenza (Mart.Cap. 567: Virgo armata, decens, rerum sapientia, Pallas / aetherius fomes, mens et sollertia fati, / ingenium mundi). L'unica fonte che definisce Minerva dea del pensiero, ma impiegando il termine greco voṽ $\varsigma$, e quindi senza alcuna velleità o valenza etimologica, è nuovamente Marziano Capella (567: rationis apex divumque hominumque sacer voũ $)$. D'altronde, a Roma esisteva una dea Mens, essa si preposta alla tutela della mente (vd. nota 36): a lei era stato dedicato 1'8 giugno del 217 a.C. da Marco Emilio Scauro, all'epoca della seconda guerra punica, un tempio sul colle capitolino (Cic. ND 2.61; Liv. 22.10.10; Fasti Venusini, Maffeiani, Tuscolani e Viae Graziosa: Degrassi, Inscr. It. XIII.2, 58, 77, 103 e 216), ma sembra figurare già anticamente tra le divinità degli indigitamenta (Aug. Civ. 4.21) e quindi di notevole arcaicità (MARBACH 1931, 936s.; HiLD 1969b, 1720); dunque, non vi era evidentemente bisogno di scindere la titolarità di un patronato così specifico, quello sulla 'mente', suddividendolo tra le sfere di competenza di più divinità. 
varroniano come fonte- ${ }^{33}$ giunge ad ipotizzare che il nome Minerva quasi quaedam Meminerva formatum est, ovvero che derivi dal verbo memini, dal momento che lei è la dea cui si affida la tutela della memoria. ${ }^{34}$ Evidentemente all'interpretazione dell'apologista cristiano non può essere concessa alcuna attendibilità scientifica, poiché tale forma non è in alcun modo documentata epigraficamente, né tanto meno è ammissibile filologicamente, ${ }^{35}$ però è innegabile che l'esistenza di tale paraetimologia sia sintomatica dell'importanza che questo aspetto doveva rivestire nel culto e nella tradizione connessa alla dea. Vi è però una seconda testimonianza in merito al patronato della nostra dea sulla memoria, anche in questo caso conservataci da uno dei Padri della Chiesa, ovvero da S. Agostino, la cui fonte è stata individuata nuovamente nelle Antiquitates Rerum Divinarum di Varrone ${ }^{36}$ queste brevi righe del De Civitate Dei, alla luce del nesso Minerva-pueritia che si è evidenziato per il minerval, acquistano certamente - come vedremo- una nuova e centrale rilevanza: in effetti, Varrone afferma che Minerva era sì la dea della memoria, ma non della memoria tout court, bensì segnatamente di quella 'dei fanciulli' (puerorum memoriam). Sant'Agostino, infatti, ebbe buon gioco nell'additare l'incoerenza e le contraddizioni della religione pagana, per la quale si era attribuita maggior importanza ad una divinità, ossia Minerva, la cui incombenza era quella di tutelare la memoria dei bambini, mentre la dea Mens, cui invece ci si rivolgeva per avere una buona mente lato sensu, era stata relegata in secondo piano ed esclusa dal gruppo degli dei selecti; al di là del chiaro intento polemico da parte dell'apologista, rimane comunque la validità dell'osservazione critica agostiniana: perché Minerva si occupava della memoria dei bambini? O meglio, ribaltando la prospettiva, perché la memoria dei bambini era talmente importante da richiedere il patrocinio di una delle divinità principali del pantheon romano e non era invece 'sufficiente', a tale scopo, l'intervento della secondaria e marginale dea Mens? Reputo che l'unica risposta plausibile sia ancora una volta nella relazione privilegiata tra Minerva ed i fanciulli romani, tra la dea ed il periodo dell'infanzia e dell'adolescenza: invero, così come la dea sovrintendeva alla formazione ed all'educazione di pueri e puellae, allo stesso modo si occupava, evidentemente, di garantire loro una buona memoria per un appropriato e consono apprendimento, in una fase sempre delicata e cruciale qual è quella della crescita.

33 Champeaux 2007, introduzione.

34 Arn. Nat. 3.31: Eandem hanc alii aetherium verticem et summitatis ipsius esse summam dixerunt, Memoriam nonnulli, unde ipsum nomen Minerva quasi quaedam Meminerva formatum est.

35 La Champeaux sostiene, erroneamente, che sia da accettare l'etimologia ipotizzata da Arnobio, per il quale Minerva deriverebbe dal sostantivo memoria (CHAmpeaux 2007, 132s.); in realtà, la tesi oggigiorno più comunemente accettata sull'origine del nome 'Minerva' è quella che risale alla radice *men-: da questa radice, infatti, la protoforma ricostruita è quella di *menes-una , la cui base etimologica è il protoindoeuropeo *menes-

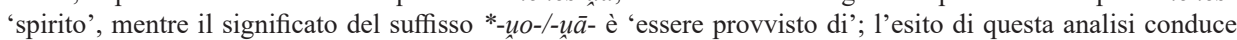
dunque alla forma femminile *menesua $\bar{a}$, di un aggettivo *menesuo- 'intelligente' (RIx 1981, 117; MezzetTi 1997-2000, 181ss.; DE VAAN 2008, 380s.). Da un punto di vista prettamente documentario, oltretutto, l'esistenza di un ipotetico Meminerva è da escludere categoricamente, essendo documentati per lo meno dal VI sec. a.C. della forma arcaica del nome della dea, ovvero quella di Menerva: le testimonianze sono sia archeologiche, dai santuari di Portonaccio di Veio (CIE 6436, 6438, 6446) e di Santa Marinella a Punta della Vipera (M. Torelli, StEtr XXXIII 1965, 505), sia letterarie, per quanto concerne una voce del Carmen Saliare riportata da Festo (Fest. 222L: Promenervat item, pro monet).

36 Aug. Civ. 7.3 (=Varro Rer.Div. fr. 135): Ibi posuerunt et Mentem deam, quae faciat pueris bonam mentem, et inter selectos ista non ponitur, quasi quicquam maius praestari homini possit. ... Quae profecto et Minervae fuerat praeferenda, cui per ista minuta opera puerorum memoriam tribuerunt. 
Ora, è ben nota la centralità che nelle società antiche rivestiva il processo di maturazione fisica e sociale, sino al conseguimento per i ragazzi dello status di adulti; un processo, inoltre, rigidamente controllato dalla comunità, a volte scandito da più riti ed iniziazioni successive ripetute, con delle tappe intermedie a cadenza ciclica - come nella Grecia classica ${ }^{37}$-, altre volte invece segnato da un'unica cerimonia puntuale, da un singolo momento che certificava definitivamente, per il giovane, il passaggio alla nuova condizione di cittadino adulto -come per di più testimoniato anche da moderni studi antropologici. ${ }^{38} \mathrm{Ed}$ anche Roma non era di certo estranea a tali rituali: invero, anche nell'Urbe, ancora in epoca storica, esistevano simili modalità di rinnovamento del corpo civico, tramite l'introduzione degli adolescenti che avevano terminato il loro percorso di maturazione sessuale e/o sociale e quindi finalmente pronti per una vita cittadina attiva: per quanto concerne i pueri, infatti, siamo ben documentati dalle fonti, le quali ricordano che, una volta compiuto il 17esimo anno di età, nel giorno dei Liberalia del 17 marzo questi deponevano la toga praetexta, l'abito dell'infanzia, e vestivano la toga virile; la cerimonia, dopo la ductio in forum dei ragazzi, terminava quindi con un sacrificio al Capitolium. ${ }^{39} \mathrm{Ma}$ la ritualizzazione di una tappa così fondamentale quale quella dell'ingresso nella comunità degli adulti, anche se non esplicitamente testimoniataci dalle fonti, esisteva anche per le puellae, come magistralmente dimostrato da Torelli alcuni decenni or sono nella circostanziata analisi effettuata prendendo spunto dagli eccezionali rinvenimenti del santuario lavinate di Minerva: ${ }^{40}$ è infatti proprio quest'ultima, insieme ad Anna Perenna, ${ }^{41}$ a sovrintendere al passaggio di status per le ragazze romane, nel quindiale compreso tra il 15 ed il 19 marzo, con le Quinquatrus, contestualmente a quello della controparte maschile, agli inizi del nuovo anno ed in corrispondenza della rinascita primaverile. $^{42}$

$37 \quad$ A Sparta le prove del rituale primitivo si erano trasformate in feste religiose ed agonistiche, ma sempre differenziate per gradi di iniziazione: le Hyakínthia, le Gymnopaidía e le Karneia; ad Atene, invece, si conserva la denominazione delle varie classi d'età: arrephoros, aletris, arktos e kanephoros (in merito vd. BRELICH 1969).

38 Vedere, in particolare, le fondamentali opere di Brelich (1969), EliAde (1980) e VAn GenNEP (1981).

39 Insieme alla toga praetexta i giovani abbandonavano anche la bulla, che insieme alla pretesta costituivano le insignia pueritiae (Pers. 2.70 e 5.31; Plu. Quaest.Rom. 101; Paul.Fest. 32L; Porph. Hor.Sat. 1.5.65). Sul tema vd. HunZiker 1969, 353; Torelli 1984, 21; Id. 1990; Massa-Pairault 1987, 218ss.; Sabbatucci 1988, 103ss.; MiLler 2002, 217ss.; Moret 2012, 92s.

40 TORELli 1984

41 Divinità arcaicissima, connessa ai rituali di rinnovamento ciclico all'inizio del nuovo anno, ma ormai di secondaria importanza nel pantheon romano in epoca tardo-repubblicana; la sua festa si svolgeva annualmente in un bosco sacro al I miglio della Via Flaminia, lungo le rive del Tevere, il 15 di marzo (Fasti Vaticani: Feriae Annae Perennae, via Flaminia ad lapidem primum; ma anche Fasti Antiates Maiores e Farnesiani-DEGRASSI, Inscr. It. XIII.2, 6, 172 e 225). Le fonti antiche ce ne parlano in relazione alla nota vicenda delle 'false nozze' in cui sono coinvolti anche Marte e Minerva, nella quale Anna Perenna si sostituisce alla nostra dea al momento delle nozze, ingannando così un innamorato Marte; le altre vicende mitografiche cui prende parte Anna Perenna sono quella relativa alla secessione della plebe sul Monte Sacro, durante la quale Anna avrebbe sfamato il popolo con i liba, ed al racconto della Anna punica, sorella di Didone, che sarebbe morta e quindi trasformatasi in ninfa nel fiume Numico (Ov. Fast. 3.145-146; 3.523-696; Sil.Ital. 8.28-241; Mart. 4.64.17; Plu. Prov.Alex. 28; Gell. 13.23.4; Macr. Sat. 1.12.6; Paul.Diac. 1.6). Sulla figura di Anna Perenna, sul suo ruolo nelle celebrazioni di marzo e per una ampia bibliografia sul tema vd. CinAGLia 2018.

42 Torelli 1984, 68ss.; Massa-Pairault 1987, 206ss.; Menichetti 1995, 84ss.; Miller 2002, 217ss.; Moret 2012, 92s. Sul tema del ciclo festivo connesso a Minerva e sulla sua primigenia ed imprescindibile funzione di dea 'del passaggio', vd. CinaGlia 2018; sulle origini e sulle celebrazioni delle Quinquatrus, festa del 'quinto giorno scuro' e legata all'arcaico calendario lunare, vd. CinAGLia 2016 b. 
Pertanto, la relazione privilegiata tra Minerva ed i pueri romani che si è evidenziata nei passi presi precedentemente in esame, con il suo intervento sulla memoria dei fanciulli e con la pratica del minerval a lei consacrato, non può essere considerata una mera supposizione: era infatti Minerva a tutelare, a vegliare, su pueri e puellae sino al momento del loro ingresso nella comunità come adulti; e se in epoca arcaica il patronato della dea sulla pueritia doveva sicuramente avere altre forme, essendo l'educazione scolastica una 'innovazione' medio-repubblicana, non può meravigliare che nella tarda repubblica e nel periodo dell'impero la crescita dei fanciulli fosse anche e soprattutto sinonimo di istruzione e che pertanto a Minerva ci si rivolgesse per avere una buona formazione e quindi anche una buona memoria: è invero noto come, nell'antichità, qualsiasi sistema di apprendimento, in particolar modo quello infantile, fosse strettamente relazionato all'imprescindibile uso della memoria; la memoria, dunque, quale emblema dell'istruzione giovanile. ${ }^{43}$ Non serve, pertanto, 'scomodare' la divinità dell'eloquenza -come sostiene la vulgata moderna- per giustificare l'intervento di Minerva nell'istruzione dei ragazzi: a lei, in quanto dea preposta alla tutela dei pueri, era infatti affidato il più ampio ed indubbiamente 'gravoso' compito di vegliare sulla crescita di ogni fanciullo, e quindi anche sulla sua formazione scolastica; d'altronde, credo che si possa comprendere solo alla luce di quanto sinora esposto l'episodio riportato da Svetonio il quale, nella vita di Caligola, ${ }^{44}$ afferma che l'imperatore, dopo aver condotto la figlia Drusilla per omnium dearum templa, Minervae gremio imposuit alendamque et instituendam commendavit: un gesto che, al di là della nota predilezione di Caligola verso la dea, testimonia come, ancora nella prima età imperiale, Minerva fosse senza dubbio alcuno la dea che presiedeva all'educazione delle puellae, sin dall'infanzia, con spiccati connotati curotrofici. ${ }^{45}$

43 Per quanto concerne il ruolo centrale della memoria nell'apprendimento, basti qui ricordare, a solo titolo di esempio, la figura dell'aedo, la cui attività di cantore-poeta si basava sull'ampio uso di un linguaggio ampiamente formulare e di topoi narrativi, 'stratagemmi' fondamentali per la trasmissione dei testi epici in forma orale che richiedeva necessariamente notevoli capacità mnemoniche.

Più in generale sulla formazione e sull'educazione dei fanciulli a Roma, vd. FraSCA 1996.

44 Suet. Cal. 25.4.

45 La Champeaux individua in questo episodio soltanto gli indizi della natura curotrofica di Minerva, parlando di 'nutrimento simbolico' ad opera della dea (CHAmpeaux 1982, 166). In realtà, il ruolo di Minerva come divinità tutelare dell'infanzia emerge chiaramente anche dall'esame dell'iconografia della dea, nello specifico di una rappresentazione mitologica riprodotta su di una cista prenestina, databile al III secolo a.C. (CIL $\mathrm{I}^{2} 563$ $=I L L R P$ 1198) e su due specchi bronzei etruschi, databili il primo alla fine del IV sec. a.C. ed il secondo tra la fine del IV e gli inizi del III (rispettivamente 1- CII 480; GerHARD, ES I, 158s.; 2 - CIE $1840=$ CII 2094; GERHARD, ES II, 275s.): il tema figurativo che accomuna questi tre oggetti, non pertinente alla mitologia greca e quindi non riconducibile alla figura di Atena, è sempre molto articolato, con la compresenza di numerose figure divine, ma al centro delle quali è costantemente rappresentata Minerva nell'atto di sostenere un Marte (Maris) bambino al di sopra di un dolio; le interpretazioni a riguardo sono molteplici, recentemente però sono state proposte delle nuove letture di tale raffigurazione, sostanzialmente assimilabili tra loro, che coerentemente hanno ampliato l'analisi dal mero rapporto mitografico tra le due divinità all'esame che questo nesso MarteMinerva ha anche a livello religioso e calendariale nel ciclo festivo romano. La Champeaux ribadisce l'idea di una Minerva curotrofica, con "soins maternels et nourriciers" verso il Marte-bambino; Torelli, invece, ha individuato nel gesto di Minerva l'atto dell'artifex nel momento della creazione di una statua - ovviamente di Marte - giustificando il rapporto tra le due divinità nella scena come il paradigma della 'nascita impossibile' del dio in riferimento alle 'nozze impossibili' tra le due divinità nel mese di marzo; successivamente la MassaPairault, accettando l'ipotesi che l'immagine di Marte sia una statua, individua nella presenza di questa sopra al pithos il momento immediatamente precedente al bagno lustrale del dio, ovvero un'allusione, vista la partecipazione di Minerva, alla lustratio dei Liberalia del 17 marzo strettamente connessa nel ciclo festivo alle Quinquatrus, concludendo che le divinità sulla cista sono "in funzione del mese di Marzo e dell'agonium 


\section{Minerva dea lanificii ${ }^{46}$}

Come abbiamo rapidamente accennato in precedenza, se il patronato della dea sull'infanzia è sicuramente una delle caratteristiche originarie del suo culto, non altrettanto si può dire del suo 'interesse' per la formazione scolastica, essendo questa stata introdotta - ed inizialmente per un numero certamente molto ridotto di studentisolo nella seconda metà del III sec. a.C. A cosa era rivolta, dunque, l'attenzione della dea? Vi erano delle attività sotto la sua tutela alle quali dovevano attendere le puellae/iniziande? Ancora una volta sono le fonti, le cui informazioni vengono integrate dai dati desunti dai rinvenimenti archeologici, ad indirizzarci verso una più che verosimile risposta a tale quesito; in questo ulteriore caso, invero, sono le numerose testimonianze che fanno di Minerva la dea degli artigiani a poter essere considerate sotto una nuova luce, ad essere passibili pertanto di una nuova lettura.

La festività principale della dea, le Quinquatrus, è indubbiamente una festa degli artigiani, secondo Verrio Flacco l'artificum dies ${ }^{47}$ e sono numerosi gli autori che si riferiscono a Minerva in questi termini e che ne fanno una divinità 'industriosa', delle 'arti' ${ }^{48}$ Ora però, quando ci accingiamo ad indagare quali corporazioni artigianali

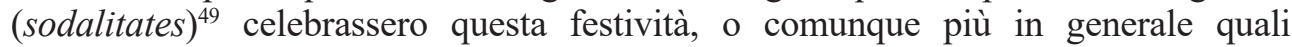
professioni vengano collegate dalle fonti al patronato della dea, non può non destare stupore la notevole disparità del numero di attestazioni per ciascuna singola categoria: come abbiamo visto in precedenza, infatti, Minerva è connessa all'insegnamento ed ai maestri (Tert. De idol. 10.1; Hier. Ep. in Ephes. 6.4; Macr. 1.12.7), un nesso che però è certamente da rovesciare in favore degli scolari, come dimostrato nella prima parte di questo lavoro; ma se escludiamo il lungo elenco ovidiano dei vari professionisti

martiale coincidente con la festa dei Liberalia"; coerentemente Menichetti, da ultimo, tirando le somme tra le numerose proposte, giunge alla conclusione che la scena riassuma "tutto il percorso iniziatico tra Marzo e Maggio, che conduce simbolicamente i giovani maschi dall'agonium Martis dei Liberalia al Mars invictus di Maggio" e che, dunque, "nel periodo compreso tra i Nominalia, cui si allude nelle raffigurazioni degli specchi, e i Liberalia, cui rimanda la scena della cista, spetta alla dea Minerva vegliare sui giovani in qualità di kourotrophos" (Champeaux 1982, 142ss.; Torelli 1986, 193s.; Massa-Pairault 1987, 201ss.; MeniChetTI 1995, 80ss. Per le precedenti interpretazione vd. Simon 1978, 138ss.; Colonna 1984, 1063 nn. 165-166-167; Hermansen 1984, 147ss.). La simbologia curotrofica è d'altronde ribadita, nuovamente da un punto di vista iconografico, dai nutriti ritrovamenti archeologici dei santuari centro-italici consacrati alla dea: invero, sia a Portonaccio di Veio, sia a Lavinium, sono numerosissimi gli ex-voto fittili, databili tra il V ed il IV secolo, rappresentanti figurine curotrofiche sedute in trono, còlte nell'atto di allattare o di abbracciare un bambino (rispettivamente Colonna - Martelli Antonioli - Martelli 2002, 198s.; Enea nel Lazio, 209ss. figg. D128D134). Quindi una Minerva che risulta già agli inizi dell'epoca repubblicana come la dea cui spetta il compito di vegliare sui giovani in qualità di kourotrophos, messa in relazione con un Maris dapprima bambino e poi ragazzo, e che quindi si rivela quale dea che accompagna il fanciullo in tutta la sua infanzia.

46 Serv. Aen. 7.805: Calathisve Minervae: Minerva et armorum dea est et lanificii.

47 Vd. nota 3.

48 Ennio (Incerta: daedala Minerva), Cesare (Gal. 6.17.1-2: Minervam operum atque artificiorum initia tradere), Frontone (Als. 3.10: Minervam artium atque artificum magistram), Ovidio (Am. 3.2.52: artifices in te verte, Minerva, manus!; Fast. 3.833: dea mille operum) e Lattanzio (Inst. 1.18.23: Sed nimirum Minerva est quae omnes repperit, ideoque illi opifices supplicant). Numerosi studiosi, anche in contributi recenti, sostengono che la tutela degli artigiani fosse addirittura l'unica competenza attribuibile a Minerva fin dalle origini, individuando nella dea romana semplicisticamente una divinità artigiana e tecnica (WISSOWA 1897, 2987; THULIN 1905, 258; LATte 1960, 165; Bailey 1975, 70-118-129; STORChi Marino 1979, 337ss.; SChIlling 1981, 85; RüPKE 1995 , 616; SCHEID 2008, 87).

49 Gaius Dig. 47.22.4. Queste corporazioni vengono nominate per la prima volta già nelle leggi delle XII Tavole, secondo le quali tali associazioni potevano stipulare al proprio interno qualsivoglia accordo, a meno che esso non violasse la legge; ma i sodales -o socii-, ovvero coloro che appartengono allo stesso collegio, secondo la tradizione risalirebbero al regno di Servio Tullio (Flor. Epit. 1.6.3). 
che il poeta esorta ad invocare la dea, ${ }^{50}$ quella dei magistri è l'unica professione esplicitamente menzionata dalle fonti in relazione a Minerva. L'unica eccezione è costituita dai fullones, sola ed effettiva corporazione cui fanno riferimento anche altri autori latini oltre al già citato passo di Ovidio: la prima fonte a riguardo è un passo di una atellana di Novio, in cui i fullones sono impegnati per l'appunto nella celebrazione delle Quinquatrus $;{ }^{51}$ in un ulteriore passo, inoltre, è Plinio il Vecchio che, trattando dei pittori e delle loro rispettive opere, relaziona nuovamente questa corporazione alla festa del 19 marzo, ricordando che il pittore Simus aveva rappresentato la bottega di un follatore nell'atto di celebrare questa giornata. ${ }^{52} \mathrm{E}$ la devozione di questo particolare gruppo di lavoratori verso Minerva è oltretutto confermata da due straordinarie testimonianze archeologiche, ovvero dal rinvenimento all'interno di due diverse fullonicae di Pompei di altrettante pitture parietali raffiguranti scene connesse alle attività dei fullones, immagini nelle quali è sempre presente la civetta, animale totemico di Atena-Minerva. ${ }^{53}$ Ma se il legame tra Minerva ed i fullones è comunque evidente

50 Ov. Fast. 3.821-832. In questa lista sono compresi fulloni, tintori, calzolai, carpentieri, medici, nuovamente i maestri, cesellatori, pittori e scultori, oltre ovviamente alla categoria dei poeti.

51 Nov. Atellanae 95-96: Plus sapiui, quin fullonem compressi Quinquatrubus. Sequere me!

52 Plin. HN 35.143: Simus iuvenem requiescentem, officinam fullonis quinquatrus celebrantem.

53 Pompei, fullonica VI, 8, 20-21 e VI, 14, 21-22 (Storchi Marino 1979, 334, nota 6; Flohr 2013, 202ss.). Ulteriore conferma di questa devozione da parte dei fullones è una iscrizione dedicatoria proveniente da Spoleto: si tratta di un'epigrafe su travertino $(58 \times 52 \mathrm{~cm})$, posta originariamente in un edificio pubblico e databile al I secolo a.C.: Minervae do[n(um) d(ant)] / fullones. / Magistri quinquen(nales) / curavere / C(aius) [F]uuli(us) C(ai) l(ibertus) Statius, / P(ublius) Oppi(us) L(uci) l(ibertus) Pilonicus, / L(uci) Magni(us) L(uci) l(ibertus) Alaucus, / Pampilus Turpili T(iti) s(ervus) $\left(C I L \mathrm{I}^{2} 2108=\right.$ CIL XI $\left.4771=I L L R P 240=I L S 3127\right)$. Questa è una delle sole tre iscrizioni dedicate a Minerva da un collegium in epoca repubblicana; infatti, i collegia la cui connessione con la dea ci è fattivamente documenta sono esclusivamente quelli dei cornicines di Roma, legati al santuario del Celio (iscrizione proveniente dagli Horti Theophilis e databile al II secolo a.C.: Miner[vae] / donum [dat] / conlegi[um cor]/nicin[um]; CIL VI 524) e dei fabri della città di Corfinium (Minervae / sacr(um) / C(aius) - Decimius / communis · lib(ertus) / Vitalis / collegio · fabror(um) / dedit; CIL IX 3148). Sui cornicines, sul loro ruolo all'interno dell'esercito e nelle cerimonie religiose vd. PotTIER 1969, 1512ss.

Assimilabili, per competenze e ruolo nelle cerimonie sacre, sono i tibicines, che intervengono il 13 giugno, alle Quinquatrus minusculae, in connessione con il culto di Minerva; e la Storchi Marino, nel più ampio discorso sul patronato della dea verso gli artigiani, arriva a sostenere che, seppur ad un "livello diverso", anche i suonatori di tibia sono degli artigiani e che per questo si giustifica la loro relazione con Minerva (STORCHI MARINO 1979, 351). Ma il rapporto tra tibicines e la nostra dea, però, non è quello di patrona-corporazione artigianale: i tibicines, invero, erano tutt'altro che una semplice categoria professionale, essendo invece espressamente preposti alle celebrazioni sacre di carattere pubblico (collegium tibicinum Romanorum qui sacris publicis praesto sunt: $C I L \mathrm{I}^{2} 988=\mathrm{VI} 3696=$ ILLRP 185; CIL I² $989=$ VI 3877) e la cui istituzione risalirebbe, secondo la tradizione, a Numa (Plu. Num. 17.3) o piuttosto a Tarquinio Prisco (Str. 5.2.2); la loro funzione era pertanto sacra, indispensabili in qualsiasi cerimonia religiosa, poiché la tibia, elaborata sul modello dell' $\alpha$ v̉ ś greco era in grado di placare gli dei (Cens. 12; Arn. 7.32 - Su tibia e tibicines vd. Vetter 1936, 808ss.; ReINACH 1969b, 300ss.; VendRIES 2004, 398 e 405ss.). Senza dubbio, invece, si configura come un rapporto di patronato quello sui fabri; a tal proposito, in effetti, si deve aggiungere che, nuovamente da una domus pompeiana (II, 8, 6: Casa del Giardino di Ercole o del Profumiere), conosciamo un affresco che raffigura alcuni uomini con un ferculum sulle spalle (Bianchi Bandinelli - Torelli 1976, Arte Romana 92; Storchi Marino 1979, 334, nota 6): su questa portantina appaiono degli artigiani, verosimilmente dei falegnami, con alle loro spalle una Minerva armata che vigila sulle loro attività; anche per i fabri, dunque, si hanno varie testimonianze relativamente al patronato di Minerva sulla loro arte.

Tirando le somme su questa parentesi epigrafica, le sole tre iscrizioni note per l'epoca repubblicana costituiscono sicuramente un numero esiguo di attestazioni rispetto alle numerose epigrafi di associazioni professionali per il medesimo periodo (per una lista aggiornata in merito vd. Diosono 2007, 26s.), con una concentrazione particolare di queste ultime tra Roma e Palestrina, anche se in realtà attestate su tutto il territorio italico ed anche in scali commerciali greci e spagnoli; questo dato risulta quanto mai degno di attenzione: è infatti curioso, e forse indicativo di una realtà più complessa di ciò che affermano comunemente le fonti, poter notare come questa dea, dipinta comunemente come patrona delle attività artigianali ed intellettuali, non sia in effetti così 
dalle fonti, appare ancor più forte il nesso tra la dea e la tessitura e la filatura: sono infatti numerose le citazioni che sottolineano questa particolare sfera di competenza, quanto queste pratiche tipicamente femminili fossero particolarmente 'care' alla dea.

Il primo autore è Properzio, il quale, narrando di Penelope e dell'inganno della tela, impiega il nome proprio della dea per indicare metaforicamente l'attività della tessitura; ${ }^{54}$ successivamente, anche Virgilio e Ovidio impiegheranno il nome di Minerva in tal senso, quest'ultimo elencando tutte le singole operazioni che competevano alle fanciulle, dalla cardatura alla filatura alla tessitura, nel raccontare la vicenda mitica delle figlie di Minia, re di Orcomeno, tramutate in pipistrelli da Dioniso poiché irato con queste per il loro disprezzo nei confronti del dio. ${ }^{55} \mathrm{Il}$ poeta mantovano, invece, descrivendo la febbrile attività da parte di Vulcano per produrre le armi con cui Enea avrebbe sconfitto Turno, accosta il lavoro notturno del dio a quello di una vedova che, per mantenere casto il letto nuziale e per allevare i propri figli, è costretta ai lavori 'minervii' durante la notte. ${ }^{56}$ E nuovamente Virgilio, ancora una volta nell'Eneide, al fine di evidenziare nel contrasto la natura combattiva di Camilla, afferma come l'eroina non fosse abituata ai lavori propri di Minerva, esaltando quindi l'operosità muliebre della dea, ma d'altro canto, al contempo rinnegandone paradossalmente il carattere guerriero ${ }^{57}$ quest'ultimo passo merita, a mio avviso, una particolare attenzione: è in effetti particolarmente significativo notare come, ormai in epoca augustea, Minerva non solo non indossi le abituali vesti da guerriera, ma che al contrario venga addirittura impiegata nel confronto con l'eroina Camilla proprio ad incarnare le virtù opposte, quelle matronali e muliebri: sulla scorta di questi versi, non è forse del tutto improbabile supporre che, ancora all'epoca di Virgilio, la Minerva guerriera, seppur inequivocabilmente presente nella concezione romana sia nel mito che nell'iconografia, avesse un ruolo secondario rispetto ad una Minerva tutelare dei lavori e delle attività femminili. ${ }^{58}$ Infine, le

ampiamente venerata, dati alla mano, dagli opifices. D'altro canto, la scarsezza della documentazione costituisce pur sempre un argomentum e silentio, e pertanto non completamente probante in merito alla diffusione della titolarità del patronato di Minerva sugli artigiani; però, al contrario, dobbiamo rilevare come una particolare venerazione da parte dei membri dei collegi professionali, per quanto riguarda Roma, fosse piuttosto riservata a Fortuna, nel suo santuario al sesto miglio della via Portuensis (Fasti Esquilini ed Amiternini - DegRASSI, Inscr. It., XIII.2, 87 e 187) e forse anche presso quello di Praeneste (CHAMPEAUX 1982, 236s.): d'altronde, era lei la divinità della 'buona sorte' - ma anche del 'caso' -, cui ci si affidava per la felice riuscita di una impresa economica (Don. Phorm. 841: «fortuna» dicta incertarum rerum, «fors fortuna» eventus fortunae bonus; Hecyr. 386: «fortuna» in incerto, «fors fortuna» in bono ponitur). Pertanto, la Minerva artium atque artificum magistra non era certamente la sola, sin dal periodo repubblicano, a sovrintendere alle attività artigianali; anzi, possiamo forse supporre che Minerva non fosse originariamente la dea universalmente preposta alla tutela degli artigiani, essendo questo compito piuttosto assegnato a Fortuna, connessa con le attività artigianali sin dalla fondazione del santuario serviano (PEDRONI 1998, 47): d'altro canto, da un punto di vista documentale, nei santuari dedicati a Minerva, per l'età repubblicana nessuna testimonianza vi è di una peculiare venerazione da parte degli artigiani (vd. in merito nota 75).

54 Prop. 2.9.5: coniugium falsa poterat differre Minerva.

55 Ov. Met. 4.32-35: [...] solae Minyeides intus / Intempestiva turbantes festa Minerva / Aut ducunt lanas aut stamina pollice versant, / Aut herent telae famulasque laboribus urgent.

56 Verg. Aen. 8.408-410: ... cum femina primum, / cui tolerare colo vitam tenuique Minerva / impositum.

57 Verg. Aen. 7.805-806: non illa colo calathisve Minervae / femineas adsueta manus. Eroina mitica a capo dei Volsci, prese parte alla guerra contro Enea.

58 Non si può certamente far risalire ad una interpretazione graeco more di Minerva questa 'incongruenza' nella sua natura: nel mondo greco, infatti, l'indole belligerante di Atena è indubbiamente connaturata alla sua primitiva natura divina, essendo ella divinità poliadica, perennemente armata e sempre accanto ai vari eroi nelle loro imprese. D'altro canto, il carattere guerriero di Minerva, o per lo meno la sua arcaicità, è seriamente posto in discussione da un distico ovidiano, nel quale si afferma che nel giorno delle Quinquatrus, ossia il dies 
ultime testimonianze sulla relazione tra Minerva e la lavorazione della lana sono quelle ricavabili dalle parole di Servio, il quale, nel commento all'Eneide, afferma che alla dea si addicono le spolette (calathis), essendo lei la dea lanificii, e che la lana è sotto la tutela di Minerva. ${ }^{59}$

D'altro canto, però, è indubitabile che la tradizione mitologica e mitografica ellenica avesse pesantemente influito sulla figura di Minerva, ${ }^{60}$ in seguito all'interpretatio greca della dea latina avvenuta sin dall'epoca arcaica; ed è altresì lecito ipotizzare, pertanto, che dietro queste vicende si possano in realtà intravvedere le 'sembianze' di Atena e del suo culto. Un'ipotesi, quest'ultima, a maggior ragione valida in considerazione del fatto che i testi sopra riportati appartengono ad opere poetiche e che i contesti in cui sono ambientati, in particolare, i brani di Properzio ed Ovidio sono evidentemente greci, il primo facendo riferimento alla vicenda di Penelope ed il secondo al mito di Dioniso e le figlie di Minia. È per di più noto che nel mondo greco classico Atena fosse coinvolta nelle attività concernenti la tessitura, sia a livello mitologico, ${ }^{61}$ sia nella pratica devozionale, in quanto patrona di coloro che lavoravano la lana: ${ }^{62}$ infatti, come afferma la Deacy, "her particular skill, woolworking, was ... the consummate female activity for a society where the proper role for women was working at the loom" ${ }^{63}$ Ma tale ruolo della donna non era certamente esclusivo della civiltà ateniese, o greca più in senso lato; sappiamo, al contrario, che tra le due virtù matronali essenziali per la donna romana, una era esattamente l'abilità nel lavorare la lana (lanificium) e numerose sono le prove documentarie a tal proposito, sia letterarie che archeologiche. ${ }^{64} \mathrm{D}$ 'altro canto, è noto che questo lavoro sia stato, fin dai tempi più remoti, ad esclusivo appannaggio delle

natalis della dea, era proibito versare sangue ed 'incrociare le spade' proprio in quanto giorno di nascita di Minerva (3.811-812: Sanguine prima vacat nec fas concurrere ferro; / causa, quod est illa nata Minerva die): è innegabile che questo divieto sia fortemente stridente se connesso ad una divinità della guerra; anche Girard, nel sottolineare questa 'incongruenza', ipotizza che "l'aspect guerrier n'était pas primordial dans l'hommage rendu a la déesse" (GIRARD 1981, 208), anche se successivamente giunge a mettere in dubbio la validità della spiegazione ovidiana (GIRARD 1989, 164). Per una probabile spiegazione di questa proibizione per il giorno delle Quinquatrus e delle sue origini, vd. Cinaglia 2018.

59 Serv. Aen. 5.284: operum haud ignara Minervae: perita lanificii; 7.805: calathisve minervae: Minerva et armorum dea est et lanificii; 8.128: aut quia et lana in tutela Minervae sit, quae pacis bellique sit cultrix.

60 In un ulteriore passo di Ovidio, non menzionato nel testo, viene descritto il celebre mito greco della sfida tra Atena-Pallade ed Aracne per stabilire chi fosse più abile nella tessitura (Ov. Met. 6.1-145).

${ }_{61}$ Nell'Iliade, la dea avrebbe indossato un vestito che lei stessa aveva cucito (5.734-735) ed in una versione della creazione dell'egida, la dea avrebbe usato la pelle del gigante Pallas per costruirla (Apollod. 1.6.2). Per le altre fonti sul rapporto tra Atena e la tessitura vd. LuYsTer 1965, 138ss.; CONSOLI 2010.

62 Secondo la descrizione del Palladio fatta da Apollodoro, ad esempio, la dea teneva la lancia nella mano destra e nell'altra fuso e conocchia (Apollod. 3.12.3); anche Pausania ci descrive una statua della dea, posta nel tempio di Erythrai, che era raffigurata con una conocchia tra le mani (Paus. 7.5.9; sulla possibilità che una simile statua esistesse anche sull'Acropoli ateniese vd. Consoli 2010, 15ss.). Nell'Antologia Palatina (ad es. 6.288 e 289) esistono vari epigrammi relativi a donne che dedicano gli strumenti della loro attività professionale di filatrici e tessitrici ad Atena (DEACY 2008, 51); ma soprattutto va ricordato che, nelle vesti di Atena Ergane, a lei viene donato durante la festa delle Panatenee il peplo che le donne di Atene tessevano insieme (BURKERT 2003, 284) e che dall'Acropoli proviene una serie di pinakes fittili, databili tra la fine del VI e gli inizi del V a.C., raffiguranti una figura femminile - probabilmente la dea stessa- nell'atto della filatura e nei quali si può riconoscere un ciclo figurativo riferibile al culto di Atena (CONSOLi 2010, 18s.).

63 DEACY 2008, 52.

64 Indicativo a questo riguardo è il noto epitaffio di Claudia, di epoca graccana, che recita: domum servavit, lanam fecit (CIL I, 2, 1211); più ampiamente sull'argomento vd. BoELS-JANSSEN 1993, 241ss. L'altra qualità basilare per le matronae romane era la castitas: la donna, infatti, doveva essere pudica ed univira; sulla documentazione letteraria ed epigrafica vd. BOELS-JANSSEN 1993, 229ss. 
donne: ${ }^{65}$ a Roma, nello specifico, sappiamo da Plinio e da Plutarco che nell'aedes di Semus Sancus sul Quirinale era dedicata una statua bronzea di Tanaquil, moglie di Tarquinio Prisco, raffigurata con fuso e conocchia nelle mani; ${ }^{66}$ Plutarco, inoltre, asserisce che Romolo avesse dispensato le Sabine da qualsiasi lavoro ad eccezione proprio di quello della lana; ${ }^{67}$ ancor più rilevante, infine, è ciò che aggiunge Plinio nel passo appena citato, sostenendo che fuso e conocchia accompagnavano la sposa nel suo trasferimento nella casa del marito. ${ }^{68}$ Queste testimonianze, dunque, non fanno altro che ribadire come, già agli esordi della storia romana, la moglie 'ideale' fosse universalmente identificata nella matrona lanifica e che, quindi, si individuassero nell'attività del lanificium particolari connotati di sacralità e simbolismo. ${ }^{69}$ Pertanto, non è da escludere a priori che le succitate fonti possano in realtà mettere in scena tradizioni e valori prettamente romani poiché, come sostiene la Boels-Janssen, gli autori latini giustificavano "cette sacralisation du travail de la laine par le traditionnel éloge des vertus du temps passé: le lanificium est pour eux signe de vertu parce qu'il rappelle la pauvreté et le labeur des temps anciens"; ${ }^{70}$ e la dimostrazione che il nesso Minerva-lanificium non sia il frutto tardivo dell'interpretatio greca o della mera influenza dei modelli letterari è, a mio avviso, il più volte citato brano di Ovidio sulle Quinquatrus.

In effetti, la seconda sezione di questo lungo passo comprende i versi destinati dal poeta ad esortare pueri e puellae affinché preghino bene Minerva: ${ }^{71}$ ma, dopo un generico incitamento ad essere docti, Ovidio chiarisce quale sia il campo di competenza della dea e cosa debbano apprendere da lei le fanciulle -e non più i pueri, si badi bene, che d'ora in avanti verranno esclusi dal discorso ovidiano: a cardare e filare la lana ed a tessere col telaio; ${ }^{72}$ soltanto nei versi successivi, invero, ossia nella

65 Già nelle tombe villanoviane della I fase (IX sec. a.C.) sono presenti fusaiole e rocchetti come segno contraddistintivo di una sepoltura femminile (Torelli 1984, 131, nota 45; Boels-JANSSEN 1993, 241).

66 Plin. HN 8.194; Plu. Quaest.Rom. 30.

67 Plu. Quaest.Rom. 15.

68 Plin. HN 8.194: inde factum ut nubentes virgines comitaretur colus compta et fusus cum stamine. ea prima texuit rectam tunicam, quales cum toga pura tironi induuntur novaeque nuptae.

${ }_{69}$ Sono invero numerose altre le testimonianze letterarie che associano l'ideale dei massimi valori muliebri al lanificium: Varro LL 5.23: Quae manu facta sunt dicam ... Panis, quod primo figura faciebant, ut mulieres in lanificio, panus / Vitr. 6.7.2: in his locis introrsus constituuntur oeci magni, in quibus matres familiarum cum lanificis habent sessionem / Ov. Am. 1.13.23-24: Tu, cum feminei possint cessare labores, / lanificam revocas ad sua pensa manum / Phaedr. 4.5.3-5: Quidam decedens tres reliquit filias: / unam formosam et oculis venantem viros; / at alteram lanificam et frugi rusticam / Colum. 12.1.9-10: Nunc vero, cum pleraeque sic luxu et inertia diffluant, ut ne lanificii quidem curam suscipere dignentur, sed domi confectae vestes fastidio sint ... Quam ob causam, cum in totum non solum exoleverit sed etiam occiderit vetus ille matrum familiarum mos Sabinarum atque Romanarum, necessaria inrespsit vilivae cura / Suet. Aug. 64.4: Filiam et neptes ita instituit, ut etiam lanificio assuefaceret vetaretque loqui aut agere quicquam nisi propalam et quod in diuturnos commentarios

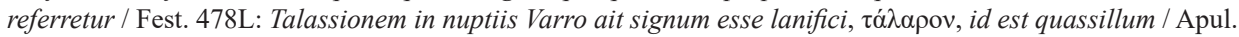
Met. 9.17.26: Tunc obstinato animo vehementer anxius Myrmex nec usquam dominam suam progredi sinebat et lanificio domestico districtam inseparabilis adsidebat / Porph. 3.12.4-5: Tibi qualum. Qualum metonymicos pro lanificio dixit. Sed mulieres per deminutionem vasculum hoc usurpant quasi illum dicentes; Serv. Aen. 4.458: haec ergo ideo a nove nuptis fiebant, ut sciret se puella domum religiosam ingredi: simul lanam ferens lanificium promittebat. Per ulteriori fonti a riguardo vd. TLL III.2, s.v. colus, 1744; VII.2, s.v. lanifica e lanificium, 929s.

70 BoELS-JANSSEN 1993, 242s.

71 Dopo l'introduzione in cui si descrivono brevemente i festeggiamenti delle Quinquatrus (vv. 809-814), vi è appunto questa seconda sezione con l'esortazione alle fanciulle (vv. 815-820); la terza parte, più estesa, è invece costituita dal precedentemente citato elenco degli artigiani devoti a Minerva (vv. 821-834); infine, un'altrettanto ampia parte finale dedicata all'origine del tempio di Minerva Capta sul Celio (vv. 835-848).

72 Ov. Fast. 3.815-820: Pallada nunc pueri teneraeque orate puellae! / Qui bene placarit Pallada, doctus erit. / 
terza sezione del brano, inizierà il noto elenco degli artigiani devoti a Minerva, lista che prende le mosse non casualmente dalla menzione di fullones e tinctores, ovvero da attività sicuramente attinenti al processo di lavorazione della lana. ${ }^{73}$ In questi versi, di conseguenza, non si possono riconoscere univocamente i tratti della greca Atena: non siamo, in effetti, in presenza della dea che invocavano le professioniste ateniesi della lana; non è una generica dea che patrocina il lavoro degli artigiani; la sua prima cura, piuttosto, come traspare dal testo ovidiano, è quella di vigilare sui fanciulli, perché a loro per primi si dirige l'esortazione del poeta. Minerva risulta, quindi, una divinità kourotrophos, cui dovevano rivolgersi le tenerae puellae al fine di acquisire quelle capacità, quelle abilità, che le avrebbero rese delle perfette matronae, mogli e madri in grado di assolvere alla funzione di guardiane della domus. ${ }^{74}$ L'elemento nuovo, sostanziale e dirimente per poter distinguere la Minerva romana dalla greca Atena, è dunque proprio quello della centralità che le fanciulle hanno nel culto della dea: è direttamente a loro che fa appello Ovidio, incitandole a pregare Minerva; e sono sempre le fanciulle, dopo aver 'placato Pallade', a doversi impratichire nelle attività connesse alla lavorazione della lana, evidentemente ancora sotto la diretta protezione della dea. Il binomio Minerva-lanificium delle fonti, pertanto, deve essere completato aggiungendo un terzo vertice a questa relazione, un vertice rappresentato per l'appunto dalle puellae ovidiane: sono loro, in effetti, che devono venerare la dea per diventare delle buone matronae apprendendo l'arte del lanificium, una delle due caratteristiche essenziali insieme alla castitas della perfetta donna romana. Un'ulteriore conferma di questo trinomio proviene dall'esame dei ritrovamenti archeologici effettuati nei due maggiori e più risalenti templi italici dedicati alla dea: infatti, nei santuari di Portonaccio di Veio e di Lavinium a Pratica di Mare sono stati recuperati in gran numero pesi da telaio, fuseruole e rocchetti, anche di dimensioni miniaturistiche, elementi fittili la cui presenza in queste aree sacre è documentata già a partire dal VI sec. a.C. ${ }^{75}$ si tratta di ex-voto chiaramente dallo scarso valore

Pallade placata lanam mollire puellae / discant et plenas exonerare colos. / Illa etiam stantis radio percurrere telas / erudit et rarum pectine denset opus.

73 La proposta della Storchi Marino, che teorizza un legame tra questi artigiani e la festività del Tubilustrium del 23 marzo, è indubbiamente da respingere; in effetti, la storica arriva a sostenere che "per i fullones e per i tinctores si può pensare ad un ruolo nella realizzazione di vestimenti particolari connessi alla produzione delle armi”" (StORChi Marino 1979, 346, nota 52) e che quindi partecipassero al Tubilustrium, giornata adibita alla purificazione delle trombe liturgiche, le tubae-dedicate a Marte-, ed alla quale partecipavano i Salii. Ma nessuna fonte letteraria, nessuna testimonianza documentaria, ci propone $-\mathrm{o}$ anche soltanto suggerisce- una ipotetica partecipazione di fullones e tinctores alle celebrazioni del 23 marzo; d'altronde, nessun artigiano era coinvolto nelle celebrazioni di questa festa, al contrario di quanto sostiene la studiosa, essendo tubicines e Salii gli unici protagonisti menzionati dalle fonti, attivi in questa giornata. Infatti, la tradizione annalistica parla di artificum dies, ovvero di 'giorno degli artigiani', e non di 'giorni degli artigiani'; il solo giorno delle Quinquatrus, dunque, era una festa -anche- degli artigiani; ma non tutto il quindiale dal 19 al 23. Il Tubilustrium, pertanto, non ha nulla a che vedere con gli artigiani in generale, e confullones e tinctores in particolare; piuttosto, come abbiamo dimostrato, il legame che vi era tra queste due corporazioni e Minerva era dovuto, molto più verosimilmente, al patronato della dea sul lanificium, attività dall'alta valenza simbolica, probabilmente connessa a Minerva sin dall'epoca arcaica.

74 Arn. Nat. 2.67: Matres familias vestrae in atriis operantur domorum, industrias suas testificantes?

75 Un catalogo dei numerosi pesi da telaio e delle fuseruole rinvenute nel santuario di Veio, presenti sin dalla prima fase dello scarico votivo databile tra VI e V sec. a.C. -materiale ascrivibile alla produzione di tipo etrusco-laziale-campano-, è in ColonNa - Martelli Antonioli - Martelli 2002, 194ss.; assolutamente da respingere le interpretazioni di tali offerte quali tributi verso una divinità tutelare degli artigiani (CIONCOLONI FERRUZZI - MARCHIORI 1989-90, 708) o piuttosto di semplici oggetti dal carattere funzionale (BAGLIONE 198990, 654), essendo la Minerva di Portonaccio venerata come divinità curotrofica e strettamente legata al mondo femminile, con funzioni salutari ed ovviamente anche come divinità guerriera, ma nessuna testimonianza 
materiale, ma al contempo sicuramente dall'alto significato simbolico e che fanno da contraltare, in forme economiche e 'popolari', alle notissime e magnifiche statue lavinati di ricche e splendide giovani offerenti, còlte nell'atto di donare alla dea $i$ simboli della loro infanzia al momento del passaggio di status: ${ }^{76}$ in effetti, se una ristretta fascia di popolazione poteva permettersi la commissione di tale coroplastica votiva di così alto pregio, ovviamente lo stesso non poteva dirsi per la maggior parte della comunità, che doveva limitarsi ad offerte molto più modiche ed economiche, quali appunto i pesi da telaio e le fuseruole, strumenti esemplari dell'artigianato tessile, della filatura e della tessitura, ma al contempo simboli inequivocabili dell'ideale della perfetta donna romana.

\section{Conclusioni}

Il lanificium, dunque, quale paradigma dell'iniziazione femminile. L'arte della lavorazione della lana quale simbolo muliebre ancestrale, per una comunità nella quale i compiti della matrona erano quelli di madre e di protettrice del focolare, di donna casta e lanifica. Ora, delineato tale quadro, dobbiamo però fare un'ultima considerazione: infatti, prendendo nuovamente a prestito le parole dell'imprescindibile lavoro della Boels-Janssen, si deve senz'altro sottolineare che "cet idéal de la matrone lanifica était l'aboutissement de la préparation au mariage dont bénéficiait la virgo sous l'égide de la pronuba, pendant la période initiatique qui suivait sa nubilité: la pronuba lui avait appris les secrets du lanificium en même temps que les mystères de l'union sexuelle"; 77 ed in effetti, nel mondo romano, ancora in epoca storica, l'esistenza dei rituali di iniziazione femminile -e l'importanza che in essi rivestiva l'apprendimento del lanificium - è indiscutibilmente certificata, per l'appunto, dalla presenza della figura della pronuba: costei, invero, interveniva in connessione con le nozze, avendo il compito di iniziare ai segreti dell'unione sessuale le puellae - nubendae; non si trattava necessariamente di una donna anziana ma, come afferma Varrone, era una donna quae ante nupsit et quae uni tantum nupta est: ideoque auspices deliguntur ad nuptias: ${ }^{78}$ le pronubae, in definitiva, erano donne che avevano raggiunto da tempo la maturità sessuale e che avevano il compito di

documentaria rivela invece un qualsiasi legame con il mondo dell'artigianato. Invece, per le testimonianze provenienti dal Santuario Orientale lavinate, documentate sin dalla prima fase del deposito votivo inquadrabile nell'orientalizzante recente (630 - 580/70 a.C.), si veda Enea nel Lazio, 218s., e FenELLi 1989-90, 494, nota 16. Il valore altamente simbolico di fuseruole, rocchetti e pesi da telaio è ribadito dalla presenza di tali oggetti in numerosi contesti deposizionali, evidentemente parte di corredi femminili, in numerose necropoli già a partire dalle fasi finali dell'Età del Bronzo e poi dell'Età del Ferro: in effetti, l'attività di fabbricazione dei tessuti, tutt'altro che oggetto di una specializzazione produttiva di tipo artigianale, nella maggior parte dei contesti era di esclusivo appannaggio delle donne, svolta esclusivamente in ambito domestico.

76 Si tratta senz'altro di uno dei più interessanti contesti votivi del Lazio antico, composto da circa un centinaio di immagini fittili di offerenti (Enea nel Lazio, 221ss. figg. D199-D264; WeIs 2014, 287ss.). Simili statue sono presenti anche a Veio, anche se in numero più ridotto e di dimensioni minori (BAGLIONE 1989-90, 657s.): l'offerta di questi ex-voto è giustificata, secondo Colonna (ColONNA - MARTELli ANTONIOLI - MARTELLi 2002, 241), dalla presenza della dea Turan, mentre per la Cioncoloni e la Marchiori queste offerte più genericamente sono attribuibili al complesso di divinità femminili presenti nel santuario, ovvero Turan ed Aritimi insieme alla stessa Minerva (Cioncoloni FerruZZI - MARCHIORI 1989-90, 710).

77 Boels-JaNSSEN 1993, 242.

78 Varro (in Serv. Aen. 4.166). Lo stesso concetto è ribadito da Festo (282L): pronubae adhibentur nuptis, quae semel nupserunt. 
iniziare le giovani ragazze alle nuova vita coniugale. Ora, è noto, anche grazie ai moderni studi antropologici, che l'iniziazione femminile, spesso portata a termine durante periodi di isolamento e segregazione della/e fanciulla/e, è sempre finalizzata alla 'rivelazione' della sessualità e della sua sacralità alla inizianda; ma è altrettanto riconosciuto che l'iniziazione non si limitasse soltanto a questo ambito, poiché, come suggerisce Eliade, proprio "durante il periodo di reclusione le novizie imparano canzoni e danze rituali, come pure certi mestieri specificatamente femminili, in primo luogo quelli dell'artigianato tessile. L'aspetto di questi mestieri è altamente simbolico: nelle fasi ulteriori di cultura lo si ritrova innalzato al rango di principio esplicativo del Mondo". ${ }^{79}$ La tessitura è infatti lavoro altamente pericoloso, cui si deve accudire nel segreto della casa: ${ }^{80}$ erano infatti le Parche a filare, e quindi a tagliare, il filo delle vite umane ${ }^{81}$ e la filatura en plein air era addirittura proibita per legge, essendo nefasta per l'agricoltura. ${ }^{82} \mathrm{Ma}$, al di là dell'enorme potere che anticamente si attribuiva alla filatura ed ai suoi strumenti, ciò che risulta fondamentale per la nostra analisi è l'evidenza della strettissima relazione tra le iniziazioni femminili, il lanificium e la sessualità; questa particolare connessione tra le puellae e le attività dell'artigianato tessile, quale emerge dai versi ovidiani, non può dunque semplicisticamente e riduttivamente essere considerata un topos letterario di matrice greca. E d'altronde, i versi in cui il poeta esorta le fanciulle a pregare la dea non hanno alcuna finalità narrativa, né tanto meno elegiaca, avendo al contrario tutto il passo sulle Quinquatrus una particolare cura descrittiva e comunque un 'sapore' marcatamente romano, quasi un rapido schizzo 'cronachistico' delle celebrazioni di tale giornata, di coloro che vi prendevano parte e, infine, del maggior luogo di culto della dea, il Minervium varroniano del Celio. ${ }^{83}$

Gli strumenti dell'artigianato tessile, pertanto, dovevano costituire per le ragazze romane inequivocabilmente degli oggetti dall'alto valore simbolico, ossia la certificazione della raggiunta nuova condizione sociale di viri potentes, ovvero in grado di prendere marito; ${ }^{84}$ al contempo, d'altronde, se il lanificium incarnava l'ideale della vita matronale, l'apprendimento di suddetta arte simboleggiava il periodo iniziatico ad essa propedeutico, la fase di preparazione della puella in vista

ELIADE 1980, 72s.

80 BoELS-JANSSEN 1993, 244s.

81 Iuv. Sat. 12.64-66: postquam Parcae meliora benigna / pensa manu ducunt hilares et staminis albi / lanificae; Fronton., De nepote amisso 2.3.14-17: Poetae autem colus et fila Fatis adsignant: nulla profecto tam sit inportuna et insciens lanifica, quae erili togae solidum et nodosum, servilei autem subtile et tenue subtemen neverit. Più in generale, sulle fonti relative alla connessione tra le Parche e l'attività della filatura, vd. TLL III.2, s.v. colus, 1744s.; VI.1, s.v. fusus, 1661; VII.2.

82 Plin. HN 28.28: pagana lege in plerisque Italiae praediis cavetur, ne mulieres per itinera ambulantes torqueant fusos aut omnino detectos ferant, quoniam adversetur id omnium spei, praecipue frugum.

83 Varro LL 5.47: [Huic iunctae] Carinae et inter eas quem locum Caeriolensem appellatum apparet, quod primae regionis quartum sacrarium scriptum sic est: Caeriolensis: quarticeps circa Minervium qua in Caelium montem itur: in tabernola est; Ov. Fast. 3.835-837: Caelius ex alto qua mons descendit in aequum, / Hic ubi non plana est sed prope plana via / Parva licet videas Captae delubra Minervae. Sulle origini e sul culto tributato alla Minerva Capta del Celio vd. CinaGlia 2016a.

84 Fest. 296L: Pubes et qui pubem generare potest. Is incipit esse a quattuordecim annis: femina a duodecim viri potens, sive patiens, ut quidam putant. L'espressione viri potens nasce come nozione tecnico-giuridica, conosciuta con certezza a partire dal I sec. d.C. e costituitasi come tale per lo meno nei due o tre secoli precedenti nell'uso della giurisprudenza, in opposizione a pubes: con il sostantivo 'pubere', invero, si indicava l'adulescens, termine che nel glossario di Festo è comunemente impiegato per indicare l'inizio dell'età adulta; lo stesso valore, pertanto, deve essere attribuito a viri potens, indicante la ragazza che aveva ormai raggiunto la maturità sessuale e quindi ormai in grado di sposarsi (TAFARO 1988, 23ss.). 
della nuova vita coniugale. Ed è proprio in questo ambito che interviene Minerva: nel sovrintendere alla crescita ed al cambiamento di condizione sociale delle ragazze, alla formazione delle puellae fino al conseguimento del loro nuovo status di viri potentes e quindi, il 23 marzo, di nubendae..$^{85}$ Ma già in epoca arcaica, la 'specializzazione' di Minerva rivolta esclusivamente al mondo femminile, al patronato sull'infanzia delle fanciulle sino alla loro completa maturità sessuale e sociale, si era ampliata ad includere sotto la sua protezione anche la componente maschile, i pueri; è esplicita ed inequivocabile, in tal senso, la presenza dell'altare della dea Iuventas nella cella di Minerva nel Capitolium: già dalla fine del VI sec. a.C., invero, la dea era ormai assurta a divinità tutelare di tutti $\mathrm{i}$ fanciulli romani, indipendentemente dal sesso - e proprio per questo inserita nella triade capitolina- ed insieme a Iuventas garante del passaggio dalla fanciullezza all'età adulta. In conclusione, dunque, tornando ai passi presi in esame, possiamo affermare che il rapporto di Minerva non era tanto con la memoria, quanto con l'educazione dei giovani romani, per i quali la memoria era semplicemente un mezzo dell'apprendimento (memoria puerorum); non con i maestri di scuola, ma con gli scolari che a lei si rivolgevano gratificandola con il dono di un simbolico obolo (minerval); non con i fullones, considerati come corporazione artigianale, ma piuttosto con le attività di cardatura, filatura e tessitura, arti paradigmatiche della condizione matronale ed apprese dalle fanciulle proprio nel corso del periodo iniziatico 'prematrimoniale' (lanificium). E se la forma smaccatamente greca della divinità di epoca classica, dall'iconografia alla mitografia, ha spesso intaccato anche la sostanza romana della dea, ancora nella tarda repubblica e nel periodo imperiale Minerva conservava ben evidenti le sue caratteristiche peculiari, di divinità indigena, soprattutto nelle forme di culto e nella devozione popolare -basti esaminare, come sopra rapidamente esposto, i depositi votivi dei santuari di epoca arcaica dedicati alla nostra dea, quali quello di Lavinium o di Portonaccio di Veio-; ma la vera e primigenia natura della dea sembra emergere, ad uno sguardo più attento, anche dalla più esigua documentazione letteraria, come abbiamo potuto constatare: Minerva, infatti, anche nelle fonti ha sempre mantenuto uno strettissimo legame con la dea Iuventas (Capitolium), connesse nel patronato sul passaggio di status, e con l'educazione e la formazione dei giovani romani (Pallada nunc pueri teneraeque orate puellae). Insomma, una Minerva meno 'versatile', per lo meno originariamente, ma indubbiamente più 'coerente', legata com'era sin dall'epoca arcaica ai riti di promozione post-puberale, competenza cui possiamo far risalire il suo patronato in epoca storica, attraverso l'influsso della greca Atena ed in seguito ad una inevitabile evoluzione cultuale nel corso dei secoli, su memoria, maestri e fullones: erano infatti formazione e lanificium, aspetti entrambi legati all'infanzia, gli ambiti su cui vigilava la dea; questo, pertanto, il filo conduttore da seguire per comprendere il percorso evolutivo di una dea dalle mille sfaccettature, ma che mai 'dimenticò' le sue originarie competenze.

85 In merito all'analisi delle successive tappe nell'iniziazione delle fanciulle romane, ai rimandi cultuali di tale processo nelle celebrazioni del mese di marzo ed alla terminologia 'giurisprudenziale' ad esso connessa, vd. CinAGLiA 2018. Una maggiore articolazione dei rituali vòlti alla celebrazione del raggiungimento della maturità fisica per le ragazze, sul modello delle feste greche, è ipotizzato dalla Weis sulla base di una attenta analisi delle varie tipologie di offerenti presenti tra gli ex-voto del santuario lavinate: la studiosa afferma, infatti, che "the Lavinium statues seem to speak of the many little celebrations and competitions" e queste, pertanto, "allow us to envision an ongoing celebration of maturation - one that for some children and adolescents, tapped for special roles in civic ritual, may have had aspects of a cursus honorum" (WeIs 2014, 307). 


\section{Bibliografia}

Baglione, M. P. (1989-90): "Considerazioni sui Santuari di Pyrgi e di Veio-Portonaccio", ScAnt 3-4, 651-667.

BaIley, C. (1975): Phases in the Religion of Ancient Rome, Westport.

Bianchi Bandinelli, R. - Torelli, M. (1976): L'arte dell'antichità classica. Etruria e Roma, Torino.

Boels-Jannsen, N. (1993): La vie religieuse des matrones dans la Rome archaïque (=Collection de l'École française de Rome 176), Rome.

Bonner, S. F. (1977): Education in ancient Rome: From the Elder Cato to the Younger Pliny, London.

Brelich, A. (1969): Paides e Parthenoi (=Incunabula Graeca 36), Roma.

BURKerT, W. (2003): La religione greca di epoca arcaica e classica, Milano.

CÈBe, J.-P. (1996): Varron, Satires Ménippées. Édition, traduction et commentaire, 11, Rome.

Champeaux, J.

(1982): Le culte de Fortuna à Rome et dans le monde romain. I. Fortuna dans la religion archaïque (=Collection de l'École française de Rome 64), Roma.

(2007): Arnobe. Contre les gentils, Paris.

Cinaglia, T.

(2016a): “Minervium vs. Minerva Capta: due facce della stessa medaglia?”, 'ILU. Revista de Ciencias de las Religiones 21, 51-78 (http://dx.doi.org/10.5209/ILUR.53840).

(2016b): "Le Quinquatrus, una festa di Minerva", Gerión 34, 145-167 (http://dx.doi. org/10.5209/rev_GERI.2016.v34.53738).

(2018): "Le Quinquatrus ed il numero 5, ovvero la correlazione tra Anna Perenna e Minerva nei rituali d'iniziazione femminile", Museum Helveticum 75/1, e.p.

Cioncoloni Ferruzzi, R. - Marchiori, S. (1989-90): "I culti del santuario di VeioPortonaccio alla luce delle testimonianze votive", ScAnt 3-4, 705-718.

CoArelli, F.

(1996): “Minerva Capta, delubra, Minervium", LTUR III, Roma, 255.

(1997-98): "Il tempio di Minerva Capta e la domus di Claudio sul Celio", RendPontAcc 70, 209-218.

Colıni, A. M. (1944): Storia e topografia del Celio nell'antichità (=Memorie della Pontificia Accademia Romana di Archeologia 7), Roma.

ColonnA, G. (1984): “Menerva”, LIMC II.1, Zurich, 1050-1074.

Colonna, G. - Martelli Antonioli, V. - Martelli, L. (2002): Il santuario di Portonaccio a Veio, 1. Gli scavi di Massimo Pallottino nella zona dell'altare (1939-1940), (=Monumenti Antichi 58, Serie Miscellanea 6.3), Roma (=Inscr. It.).

Consoli, V. (2010): “Elmo, fuso e conocchia. Per un'iconografia di Atena Ergane”, Eidola VII, 9-28.

DeACY, S. (2008): Athena (=Gods and Heroes of the Ancient World VII), London-New York.

Degrassi, A. (1963): Inscriptiones Italiae. Vol. XIII - Fasti et Elogia. Fasciculus II - Fasti anni numani et iuliani, Roma.

Diosono, F. (2007): Collegia. Le associazioni professionali nel mondo romano (=Arti e mestieri nel mondo romano antico 1), Roma.

Dubosc, G. (2002): “Minerve et l'argent”, Pallas 60, 381-393.

Eliade, M. (1980): La nascita mistica. Riti e simboli d'iniziazione (=Le scienze umane), Brescia. 
Enea nel Lazio (1981): AA.VV., Enea nel Lazio. Archeologia e mito: bimillenario virgiliano. Catalogo della mostra. Roma 22 settembre - 31 dicembre 1981, Campidoglio, Palazzo dei Conservatori [Mostra], Roma.

Fenelli, M. (1989-90): “Culti a Lavinium: le evidenze archeologiche”, ScAnt 3-4, 487-505.

Flohr, M. (2013): "Ulula, Quinquatrus and the occupational identity of fullones in early imperial Italy”, [in] M. Gleba - J. Pásztókai-Szeöke (eds.), Making textiles in pre-Roman and Roman times. People, places, identities (=Ancient Textiles Series 13), Oxford, 192207.

Fougères, G. (1969): “Minerva”, DAGR, III.2, Graz, 1910-1930.

FrascA, R. (1996): Educazione e formazione a Roma. Storia, testi, immagini (=Storia e Civiltà 41), Bari.

Frazer, J. G. (1976): Ovid Fasti, London.

Gatti lo Guzzo, L. (1978): Il deposito votivo dall'Esquilino detto di Minerva Medica (=Studi e materiali di Etruscologia e Antichità Italiche 17), Firenze.

Gerhard, E. (1840-97): Etruskiche Spiegel, Berlin, 5 vols.

Giannelli, G. (1993): “Caelius Mons”, LTUR I, Roma, 208-211.

GIRARD, J. L.

(1981): "La place de Minerve dans la religion romaine au temps du principat", $A N R W$ II.17.1, Berlin, 202-232.

(1989): "Minerva Capta: entre Rome et Faleries", REL 67, 163-169.

Granino Cecere, M. G. (2001): “Quinquatrus. Tradizione popolare e tradizione antiquaria di una festività del calendario romano", ŽivaAnt 51, 25-38.

Guirard, C. (1997): Varron. Économie Rurale. Livre III, Paris.

Hermansen, G. (1984): “Mares, Maris, Mars, and the archaic gods”, StEtr 52, 147-164.

HiLD, J. A.

(1969a): “Juventas, Juventus", DAGR III.1, Graz, 785-786.

(1969b): "Mens", DAGR III.2, Graz, 1720.

(1969c): “Quinquatrus", DAGR IV.1, Graz, 802-804.

Hunziker, R. (1969): “Toga. Prise de la toge virile”, DAGR V, 347-353.

Jordan, H. - Huelsen, C. (1970-71): Topographie der Stadt Rom im Alterthum, Roma, 2 vols.

Jullian, C. (1969): “Juvenes, Juventus”, DAGR III.1, Graz, 782-785.

LATte, K. (1960): Römische Religionsgeschichte (=Handbuch der Altertumswissenschaft V.4), München.

LUYSTER, R. (1965): "Symbolic elements in the cult of Athena", HR 5, 133-163 (http://dx.doi. org/10.1086/462518).

MarbaCH, E. (1931): “Mens", RE XV.1, Stuttgart, 936-937.

Massa-Pairault, F. H. (1987): “De Préneste à Volsinii: Minerve, le fatum et la constitution de la société", ParPass 42, 200-235.

Menichetti, M. (1995): ... Quoius forma virtutei parisuma fuit ... Ciste prenestine e cultura di Roma medio-repubblicana, Roma.

Mezzetti, N. (1997-2000): “Origini del nome e del culto di Minerva”, AnnPerugia 33, 173 191.

Miller, J. F. (2002): “Ovid's Liberalia”, [in] G. Herbert-Brown (ed.), Ovid's Fasti. Historical Readings at its Bimillennium, Oxford, 199-224 (http://dx.doi.org/10.1093/ acprof:oso/9780198154754.003.0009). 
Montanari, E. (1988): Identità culturale e conflitti religiosi nella Roma repubblicana (=Filologia e Critica 54), Roma.

Moret, J.-M. (2012): “Le feste dei nani”, [in] C. Bocherens (ed.), Nani in festa. Iconografia, religione e politica a Ostia durante il secondo triumvirato (=Bibliotheca Archaeologica 26), Bari, 49-108.

Pavolini, C. (1993): “Caput Africae”, LTUR I, Roma, 235.

Pedroni, L. (1998): “Ipotesi sull'evoluzione del calendario arcaico di Roma”, PBSR 66, $39-55$.

Platner, S. B. - Ashby, T. (1965): A Topographical Dictionary of Ancient Rome, Roma (http://dx.doi.org/10.1017/CBO9781316219706).

Pottier, E. (1969): “Cornu, Cornus”, DAGR I.2, Graz, 1510-1514.

ReINACH, A. (1969): “Tibia”, DAGR V, Graz, 300-342.

Richardson, L. (1992): A New Topographical Dictionary of Ancient Rome, Baltimore.

RIx, H. (1981): "Rapporti onomastici fra il pantheon etrusco e quello romano", [in] AA.VV., Gli Etruschi e Roma. Incontro di studi in onore di Massimo Pallottino. Roma 11-13 dicembre 1979, Roma, 104-126.

RüPKE, J. (1995): Kalender und Öffentlichkeit, Die Geschichte der Repräsentation und religiösen Qualifikation von Zeit in Rom (=Religionsgeschichtliche Versuche und Vorarbeiten 40), Berlin-New York.

SabBatucci, D. (1988): La religione di Roma antica: dal calendario festivo all'ordine cosmico (=La Cultura 67), Milano.

SCHEID, J. (2008): "Il culto di Minerva in epoca romana e il suo rapporto con la Minerva di Travo", [in] AA.VV., Minerva Medica in Valtrebbia. Scienze storiche e scienze naturali alleate per la scoperta del luogo di culto. Atti del Convegno tenutosi il 7 ottobre 2006 in Travo (PC), (=Quaderni di archeologia dell'Emilia Romagna 19), Piacenza, 85-91.

SCHILLING, R.

(1981): “Les découvertes de Lavinium", ParPass 36, 84-86.

(2003): Ovide. Les Fastes, I, Paris.

Schürmann, W. (1985): Typologie und Bedeutung der stadtrömischen Minerva-Kultbilder (=Rivista di Archeologia suppl. 2), Roma.

Scullard, H. H. (1981): Festivals and Ceremonies of the Roman Republic (=Aspects of Greek and Roman Life), London.

Simon, E. (1978): “Il dio Marte nell'arte dell'Italia centrale”, StEtr 46, 135-147.

Storchi Marino, A. (1979): “Artigiani e rituali religiosi nella Roma arcaica”, RendNap 54, 333-357.

Tafaro, S. (1988): Pubes e Viripotentes nella esperienza giuridica romana, Bari.

Thulin, C. (1905): "Minerva auf dem capitol und Fortuna in Praeneste", RheinM 60, 256261.

TORELLI, M.

(1984): Lavinio e Roma. Riti iniziatici e matrimonio tra archeologia e storia, Roma. (1986): "La religione", [in] G. Pugliese Carratelli (ed.), Rasenna. Storia e civiltà degli Etruschi, Milano, 157-237.

(1990): "Riti di passaggio maschili di Roma arcaica", MEFRA 102, 93-106 (http://dx.doi. org/10.3406/mefr.1990.1661).

Valentini, R. - Zucchetti, G. (1940-41): Codice topografico della città di Roma, Fonti per la storia d'Italia, I; Curiosum Urbis Romae regionum XIII, II, Roma. 
DE VAAN, M. (2008): Etymological Dictionary of Latin and the other Italic Languages (=Leiden Indo-European Etymological Dictionary 7), Leiden-Boston.

VAN GenneP, A. (1981): I riti di passaggio, Torino.

VENDRIES, C. (2004): "Musique romaine", ThesCRA 2, 397-415.

Vetter, W. (1936): “Tibia”, RE VIA.1, Stuttgart, 808-812.

WEIS, A. (2014): "The public face of girlhood at Latin Lavinium in the $4^{\text {th }}-3^{\text {rd }}$ centuries B.C.E.", [in] S. Moraw - A. Kieburg (eds.), Mädchen im Altertum. Girls in antiquity (=Frauen, Forschung, Archäologie, 11), Münster, 287-307.

Wissowa, G. (1897): “Minerva”, [in] W. H. Roscher, Ausführliches Lexikon der griechischen und römischen Mythologie, II.2, Leipzig, 2982-2992.

ZIEBARTH, E. (1919): “Iuvenes”, RE X.2, Stuttgart, 1358. 